\title{
Joint Admission Control and Power Allocation in Hospital Networks based on Cognitive Radios
}

\author{
by

\section{Yicong Liu} \\ B.Sc., China University of Geosciences, Beijing, China, 2011
}

\author{
A Thesis \\ Presented to the School of Graduate Studies at \\ Ryerson University \\ in partial fulfilment of the \\ requirements for the degree of \\ Master of Applied Science \\ in the Program of Electrical and Computer Engineering
}

Toronto, Ontario, Canada, December 2013

(C) Yicong Liu 2013 


\section{Author's Declaration}

I hereby declare that I am the sole author of this thesis.

I authorize Ryerson University to lend this thesis to other institutions or individuals for the purpose of scholarly research.

Author's Signature:

I further authorize Ryerson University to reproduce this thesis by photocopying or other means, in total or in part, at the request of other institutions or individuals for the purpose of scholarly research.

Author's Signature: 


\title{
Abstract \\ Joint Admission Control and Power Allocation in Hospital Networks based on Cognitive Radios
}

(C) YICONG LIU, 2013

\author{
Master of Applied Science \\ Computer Networks \\ Ryerson University
}

In this thesis, we present an approach to solve the joint call admission control and power allocation problem in a hospital environment based on cognitive radio. Specifically, a multi-objective non-convex mixed integer non-linear programming (MINLP) problem with weighted-sum method for wireless access in an indoor hospital environment has been formulated in order to maximize the number of admitted secondary users and minimize transmit power while guaranteeing the throughput of all secondary users and satisfying the interference constraints for the protected and primary users. To solve this MINLP problem with different weights given to different objectives, we propose to use the standard branch and bound algorithm as appropriately modified to find the optimal solution. We also coded a specific program using OPTI Toolbox to find the minimum objective function value, number of admitted secondary users and all related values such as total system power and throughput. To analyze the numerical results, we considered three cases with equal and non-equal weights. We also changed the values of interference and maximum source power to obtain and analyze different results comparing with the normal one. Our results indicate that more power is allocated and better throughput is guaranteed while the number of admitted users is increasing. However, as they increase, the objective function value increases steadily as well, which means that it is more difficult to reach our minimizing objective. 


\section{Acknowledgement}

I would like to express my sincere gratitude to my supervisor, Prof. Alagan Anpalagan for his continuous encouragement and support. He was always generous with his invaluable time and always motivating me and teaching me by example how to become competent and professional. It was a great privilege to work with him.

I would like to acknowledge the Department of Electrical and Computer Engineering and the School of Graduate Studies at Ryerson University for their support in terms of financial aid, and work experience as a graduate assistant.

I would also like to thank my defense committee for taking the time and effort to review my work and provide me with their insightful comments.

Thanks are also due to my colleagues in the WAN group present and past, I am lucky to be a part of this group where a team spirit truly prevails. I would especially like to thank Dr. Muhammad Naeem and Nick Majedi for their help in the early stages of this work.

I can never find the words to thank my wife, Yi Cai, who was amazingly patient and understanding and who helped me through the most difficult times of my life. She not only believed in me and was always supportive but she also offered me her advice and her valuable help whenever I needed it.

My special thanks goes to my parents, especially my mother who is always a great source of love and motivation, I could never thank her enough for everything she has done for me. I would also like to thank my parents in-law and my siblings for their love and encouragement. 


\section{Contents}

1 Introduction 1

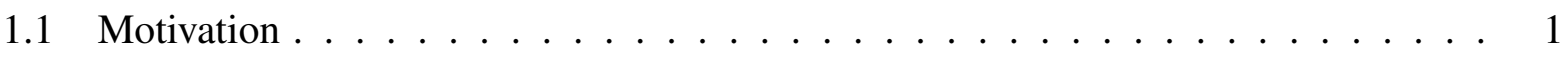

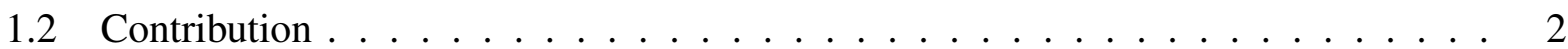

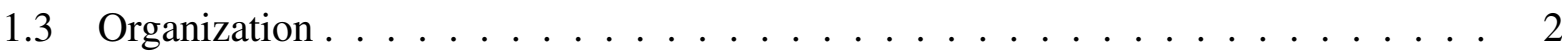

2 Review of e-Healthcare $\quad 4$

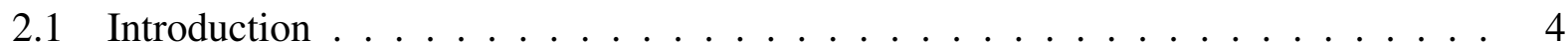

2.2 e-Healthcare services and elements . . . . . . . . . . . . . . . 4

2.3 Wireless technologies and standards . . . . . . . . . . . . . . . 10

2.4 Potential challenges in design and implementation . . . . . . . . . . . . . 16

2.4 .1 Power allocation $\ldots \ldots \ldots \ldots \ldots \ldots$

2.4 Resource management . . . . . . . . . . . . . . . 17

2.4 .3 QoS support . . . . . . . . . . . . . . . 17

2.4.4 Emerging technologies . . . . . . . . . . . . . . 20

2.5 Chapter Summary . . . . . . . . . . . . . . . . . . . . . . 23

3 Communication Network Model in Hospital 24

3.1 Communication Network in Hospital . . . . . . . . . . . . . . . . . . . . . 24

3.2 Weighted Resource Maximization Problem _ . . . . . . . . . . . . . . . . 29

3.3 Mixed Integer Non-linear Program . . . . . . . . . . . . . . . . . . . 32

3.4 Proposed Branch-and-Bound Algorithm . . . . . . . . . . . . . . . . . 36 
3.5 Chapter Summary . . . . . . . . . . . . . . . . . . . . . . . 40

4 Simulation and Results $\quad 42$

4.1 Value Assignment . . . . . . . . . . . . . . . . . . . 42

4.2 Results . . . . . . . . . . . . . . . . . . . 45

4.2 .1 Equal Weight Case . . . . . . . . . . . . . . . . 47

4.2.2 Unequal and Dominant Weight Case . . . . . . . . . . . . . . . . . 51

4.2.3 Unequal and Non-dominant Weight Case . . . . . . . . . . . . . . . 55

4.3 Chapter Summary . . . . . . . . . . . . . . . . . . . . . 61 61

5 Conclusions and Future Work $\quad 62$

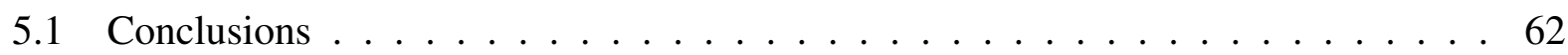

5.2 Future work . . . . . . . . . . . . . . . . . 63 


\section{List of Figures}

2.1 Some of the elements of e-Healthcare. . . . . . . . . . . . . . . . 6

2.2 Services of e-Healthcare . . . . . . . . . . . . . . . . . 7

2.3 Typical e-Healthcare devices. . . . . . . . . . . . . . . . . 11

2.4 Wireless technologies used in wireless e-Healthcare systems . . . . . . . . . . 12

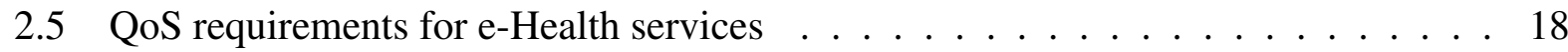

3.1 Network model . . . . . . . . . . . . . . . . . . . . 25

3.2 Network interference model . . . . . . . . . . . . . . . . . 27

3.3 Flow Chart . . . . . . . . . . . . . . . . . . . . 41

4.1 A single-floor system model $\ldots \ldots \ldots \ldots \ldots$

4.2 Number of admitted users when $I_{m}$ changes $\ldots \ldots \ldots \ldots \ldots$

4.3 Number of admitted users when $P_{s}$ changes $\ldots \ldots \ldots$. . . . . . . . 47

4.4 Total transmitting power (Watt) of all secondary users when $I_{m}$ changes $\ldots$. . . . 49

4.5 Total transmitting power (Watt) of all secondary users when $P_{s}$ changes . . . . . 49

4.6 Function value when $I_{m}$ changes $\ldots \ldots \ldots \ldots \ldots$

4.7 Function value when $P_{s}$ changes $\ldots \ldots \ldots \ldots$. . . . . . . . . . . 49

4.8 Throughput (bps) of all secondary users when $I_{m}$ changes $\ldots \ldots \ldots$

4.9 Throughput (bps) of all secondary users when $P_{s}$ changes $\ldots \ldots$. . . . . . . 50

4.10 Number of admitted user when $I_{m}$ changes $\ldots \ldots \ldots \ldots \ldots$

4.11 Number of admitted user when $P_{s}$ changes $\ldots \ldots \ldots \ldots \ldots$ 
4.12 Total transmitting power (Watt) of all secondary users when $I_{m}$ changes . . . . . 52

4.13 Total transmitting power (Watt) of all secondary users when $P_{s}$ changes $\ldots$. . . . 52

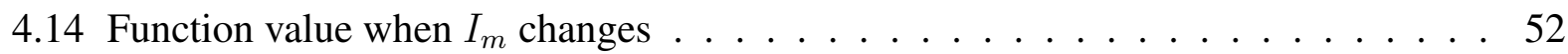

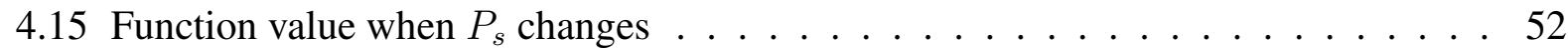

4.16 Throughput (bps) of all secondary users when $I_{m}$ changes $\ldots \ldots \ldots$

4.17 Throughput (bps) of all secondary users when $P_{s}$ changes $\ldots \ldots$. . . . . . . 53

4.18 Number of admitted user when $I_{m}$ changes $\ldots \ldots \ldots$. . . . . . . . 54

4.19 Number of admitted user when $P_{s}$ changes . . . . . . . . . . . . . . . 54

4.20 Total transmitting power (Watt) of all secondary users when $I_{m}$ changes . . . . . 54

4.21 Total transmitting power (Watt) of all secondary users when $P_{s}$ changes $\ldots$. . . . 54

4.22 Function value when $I_{m}$ changes $\ldots \ldots \ldots \ldots \ldots$

4.23 Function value when $P_{s}$ changes $\ldots \ldots \ldots \ldots$

4.24 Throughput (bps) of all secondary users when $I_{m}$ changes $\ldots \ldots \ldots$

4.25 Throughput (bps) of all secondary users when $P_{s}$ changes $\ldots \ldots \ldots 5$

4.26 Number of admitted user when $I_{m}$ changes $\ldots \ldots \ldots \ldots$

4.27 Number of admitted user when $P_{s}$ changes $\ldots \ldots \ldots \ldots$

4.28 Total transmitting power (Watt) of all secondary users when $I_{m}$ changes $\ldots$. . . . 56

4.29 Total transmitting power (Watt) of all secondary users when $P_{s}$ changes $\ldots \ldots$. . . 56

4.30 Function value when $I_{m}$ changes $\ldots \ldots \ldots \ldots \ldots$

4.31 Function value when $P_{s}$ changes $\ldots \ldots \ldots \ldots$. . . . . . . . . . 57

4.32 Throughput (bps) of all secondary users when $I_{m}$ changes $\ldots \ldots$. . . . . . 57

4.33 Throughput (bps) of all secondary users when $P_{s}$ changes $\ldots \ldots \ldots$. . . . . 57

4.34 Number of admitted users . . . . . . . . . . . . . . . . . . . 58

4.35 Total transmitting power (Watt) of all secondary users . . . . . . . . . . . . 58

4.36 Function value $\ldots \ldots \ldots \ldots \ldots \ldots \ldots$

4.37 Throughput (bps) of all secondary users $\ldots \ldots \ldots \ldots$

4.38 Number of admitted users . . . . . . . . . . . . . . . . . . . . . . 59 
4.39 Total transmitting power (Watt) of all secondary users . . . . . . . . . . . . . 59

4.40 Function value . . . . . . . . . . . . . . . . . . . 59

4.41 Throughput of (bps) all secondary users . . . . . . . . . . . . . . 59

4.42 Number of admitted users . . . . . . . . . . . . . . . . . . . . . . 60

4.43 Total transmitting power (Watt) of all secondary users . . . . . . . . . . . . . 60

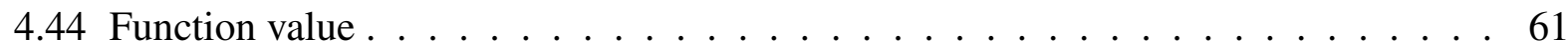

4.45 Throughput of $(\mathrm{bps})$ all secondary users $\ldots \ldots \ldots$. . . . . . . . . . . . 61 


\section{List of Tables}

3.1 Key Notations . . . . . . . . . . . . . . . . . . . . . . 28

4.1 Notational Values . . . . . . . . . . . . . . . . . . . . . . . . 43

4.2 User Configuration . . . . . . . . . . . . . . . . . . . 47 


\section{Chapter 1}

\section{Introduction}

\subsection{Motivation}

Cognitive radio system is an emerging wireless communication system which can adaptively detect the operating radio parameters (e.g., waveform, protocol, operating frequency, and networking) [1] and use the frequency spectrum efficiently. It is designed to use the best wireless channels in its vicinity such that a radio automatically detects available channels in wireless spectrum, then accordingly changes its transmission or reception parameters to allow more concurrent wireless communications in a given spectrum band at one location. This technology makes it possible for users with different priorities to utilize one spectrum at one location without causing harmful interference to each other. Thus, with cognitive radio, we can avoid EMI (Electro-Magnetic Interference) to both medical devices and users in a hospital environment in order to keep all medical devices working properly and also keep all patients in the hospital healthy.

As for EMI, power is a key factor that affects the level of harmness. Wireless networks in a hospital environment consume different amount of power, which depends on how many medical devices are transmitting, which communication protocols and power control methods are being used, and what kinds of electronic medical devices are being used by both of servers and users [2]. In this thesis, we try to do power control because it is an effective way to reduce EMI and increase 
the capacity of the wireless network in the hospital environment [3].

Since power and interference level are both controlled, we also do call admission control to reduce power consumption and EMI level further on. In recent years, many e-Healthcare applications with high media quality are emerging which needs high QoS (Quality of Service) [4]. Admission control becomes necessary for us to keep the minimum QoS for admitted users by allowing only a small portion of users in a hospital to transmit at one time.

Besides doing admission control and power control, we also guarantee QoS performance of all secondary users while maximizing the system throughput of all secondary users. To guarantee the throughput is a must since the network becomes very congested which leads to throughput degradation. This issue will be harmful to eHealthcare applications and users who demand high reliability and priority, especially when HD (high definition) images and videos are transmitting through the wireless network in a hospital.

\subsection{Contribution}

The key contributions of this thesis can be summarized in the following points:

- formulating the optimization problem taking three conflicting factors into account, including admission control, power allocation control, throughput guarantee,

- solving the problem using branch-and-bound algorithm, coding a MATLAB program to obtain the optimal results with OPTI Toolbox,

- numerically analyzing the impact of different weights on each of these factors and providing wireless network design guidelines.

\subsection{Organization}

In this thesis, we first give an overview of telecommunication integration in e-Healthcare including technologies, applications and challenges in recent years. Secondly, we design our system model 
which is a single floor of a hospital with doctors, nurses, patients, and medical devices. Next, we combine admission control and green power allocation concept in our formulation and propose a basic branch and bound algorithm to solve it using OPTI Toolbox in MATLAB. We further provide numerical results calculated by our program to determine the optimal solution. Finally, we provide the main conclusion and future works of this thesis. 


\section{Chapter 2}

\section{Review of e-Healthcare}

\subsection{Introduction}

e-Healthcare is the integration of digital data processing, computing and communication technology into the traditional healthcare services [5]. One of the important characteristics of e-Healthcare is that patient data must be available to caretakers in real-time manner without the constraints of time and location. This is referred to as pervasive health monitoring $[6,7]$.

With the continuous growth of elderly population, there has been a rise in need for more cost effective and reliable healthcare services that can provide both patients and caretakers with continuous monitoring, appliance control, medical data access, memory enhancement, emergency communications, etc. In the past decade, various research has been conducted in integrating wireless communication technologies, artificial intelligence and sensors into medical devices [8].

\section{2 e-Healthcare services and elements}

With the advancement of medical devices and integration of communication and computing systems with such devices, the whole world can be considered as an interconnected village. For example Figure 2.1 summarizes some of the services of e-Health and illustrates the integrity of these services within a typical hospital, home and tele-health environment. Considering hospital 
as the center of health services, within a hospital there are several doctors, caregivers, diagnostic and monitoring devices. The data from these devices or from reports of the doctors/caregivers are stored in a local database, synchronized with a central eHR database. Patients have various sensors attached to them. Some of them acquire vital data and have to transmit in real-time. Other than patients, rooms and hospital beds have different sensors from proximity sensors to occupancy and pressure detectors. These sensors and transmitters constantly send their accumulated data to the controllers which are connected to the central monitoring room. With the improvement of wireless transmission systems, patient monitoring and tele-health services are now possible in remote locations. Authors in [9] also mention other benefits such as lowering the response time of caregivers in emergencies, improving the life quality of elderly, enabling the large-scale filed studies of human behavior, and early detections as a result of real-time monitoring of patients. Sometimes there is need to provide emergency services in large disaster areas [9]. In such cases a field hospital would be set up in the locations with a large number of injured people. Infrastructure-less wireless nodes and sensors are then used to acquire help from remote doctors and access patients' eHR (electronic health record).

There is a wide variety of services offered under e-Healthcare framework. Figure 2.2 summarizes these services. As can be noted from the summarized list, there have been lots of research activities over the last decade in computerized e-Health service delivery mechanisms with the use of ICT technology. In the following we discuss them.

Monitoring patients consists of pre-hospital monitoring, in-hospital monitoring, and pervasive health monitoring. Pre-hospital monitoring usually implies observing patients on the way to hospital. This is done inside equipped ambulances and helicopters in some cases. Depending on the technology of use, these vehicles might have different capabilities including tools for electrocardiography, cardiac dysrhythmia detection, blood pressure monitoring and defibrillation pads. In-hospital monitoring in eHealthcare comprises vital signs monitoring, electrocardiography, capnography, child birth monitoring, blood pressure, glucose and flow monitoring, body temperature monitoring, etc. Some of the used devices for these purposes transmit realtime wireless data 


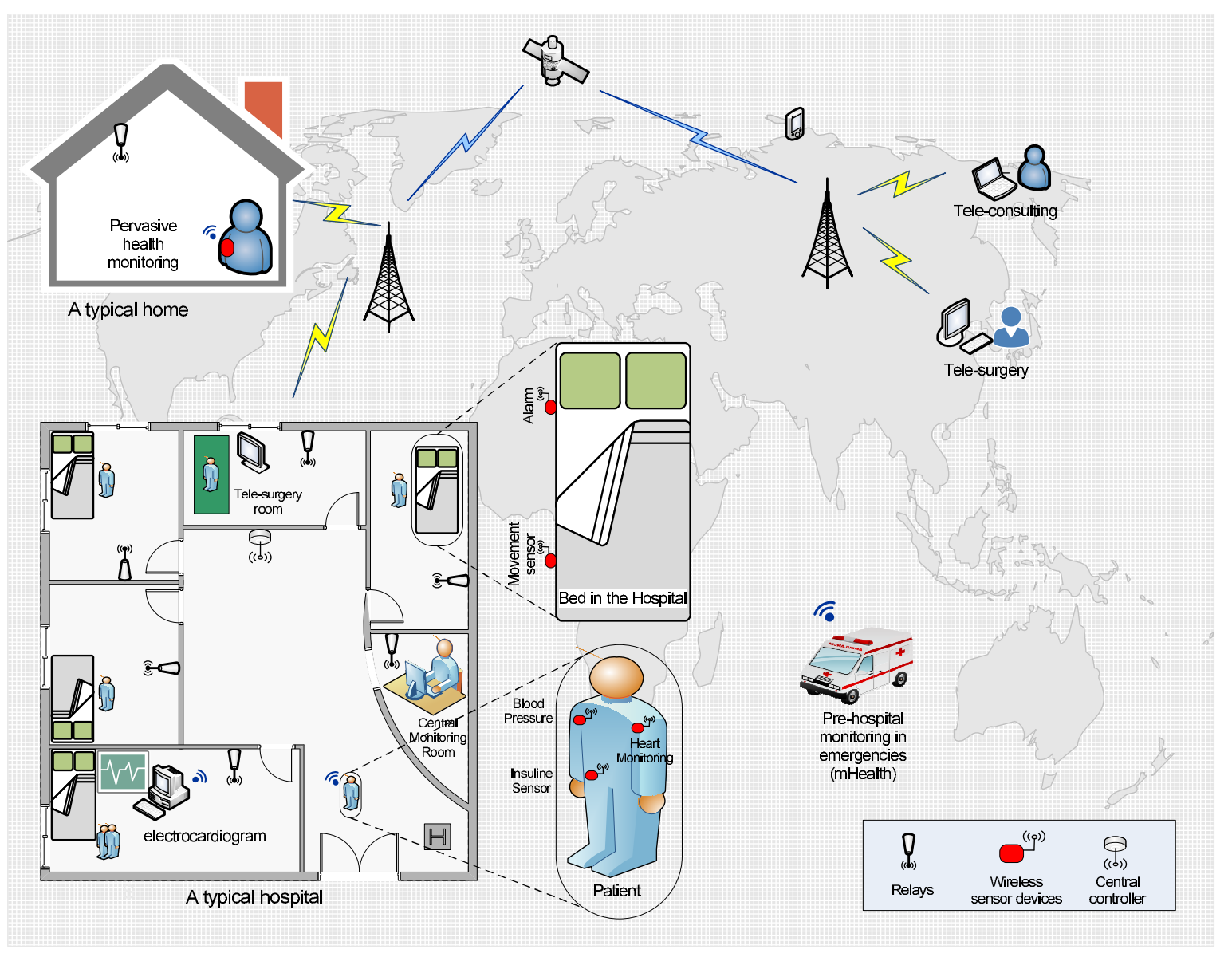

Figure 2.1: Some of the elements of e-Healthcare. 


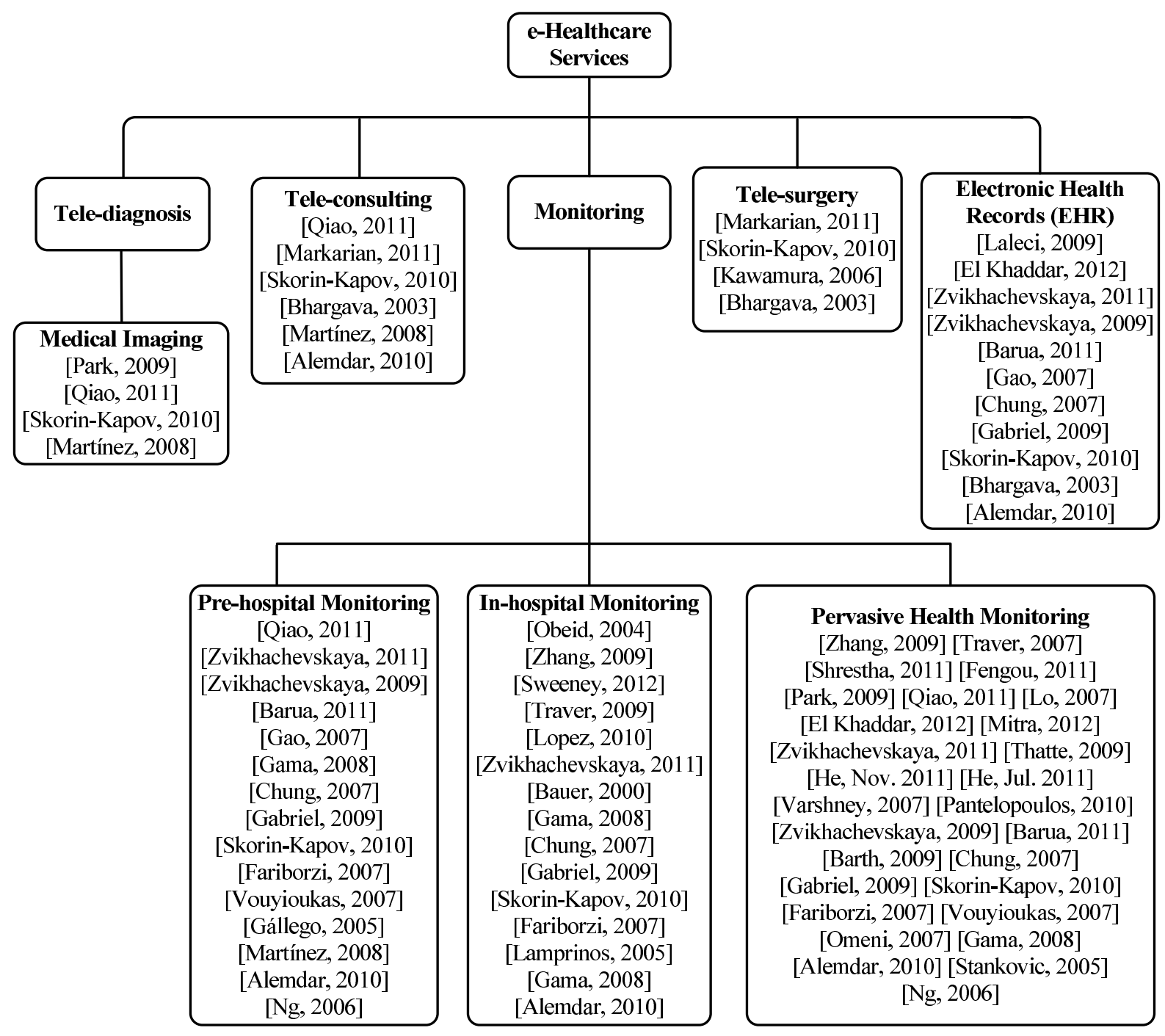

Figure 2.2: Services of e-Healthcare.

which should access the channel whenever they have data available, such as telemetry devices.

Pervasive health monitoring is an important and fast-growing topic in eHealthcare which improves prevention and early detection of diseases. One of the biggest challenges in pervasive health monitoring is quality of service $(\mathrm{QoS})$ as data streams from various sensors have different priorities and an extra delay in high priority packets may incur fatal consequences [10]. In [6], authors investigate different applications, requirements and challenges of pervasive healthcare. Wireless infrastructures are evolving constantly, thus improving the quality of transmission and coverage in existing and emerging healthcare applications, lowering the stress on caregivers and reducing 
the overall cost of health services. For instance, patients' medical information can be accessed anytime and anywhere using wireless networks and appropriate devices. It has been reported in [6] that pervasive health monitoring eliminates the restraints of time and location and that patients would receive help much faster whenever they need it. Context-aware intelligence in pervasive services would reduce false alarms. As described in [11], one of the major applications of pervasive health monitoring is in-home monitoring. As the population of world ages more and more, the need to taking care of elderly increases. Exploiting the capabilities of eHealthcare other than making available the health status information of the patients [8], would help such patients to control the appliances, equipments and tools at home, to remember to take their drugs, to look up medical information and to communicate with caregivers when necessary and in emergencies. According to [8], other applications of in-home pervasive healthcare are fall/movement detection, location tracking and medication intake monitoring. Integrating intelligent medical microsensors into wireless communication networks improves the life quality by providing complete observation of patients without affecting their normal life which causes lowering of the costs as well [12]. High speed wireless communications enable real-time monitoring of neural signals which can be used in implantable and wearable wireless sensors. This will be helpful for patients suffering from paralysis, blindness or deafness [13].

Wireless personal area networks (WPANs) such as ZigBee networks can be used to develop a home health monitoring platform in which the devices are mostly non-identical in terms of data rate and/or packet size. In such a network, the IEEE 802.15.4 MAC can be used in beacon-enabled mode, which supports guaranteed time slot allocation for time-critical data transmissions [14]. Authors in [15] propose a new frame work architecture for low cost personalized e-Health services in which standardized services are deployed over next generation IP networks. At a smart home environment, tele-monitoring consists of continuous bio-sensing of patient, context-sensing of the living conditions, dynamic creation of the appropriate group of eligible subject (e.g. doctor, relative, nurse) to deal with the situation, and transmission of information or alarm signals to the eligible subjects [15]. In [16], tele-monitoring is described as transmission of a patient's 
vital bio-signals and other related data which are used in treating chronic diseases or post-hospital home care. This may involve multi-parametric signal transmission including ECG, blood pressure, saturation of peripheral oxygen, glucose level, patient's activity, air temperature, humidity and air pressure.

Authors of [17] have provided an overview of development of body sensor networks (BAN) which are proposed to improve patient care, manage chronic diseases, promote lifelong health for elderly and maintain a truly pervasive monitoring and sensing environment. Authors in [7] authors present wearable wireless body area networks (WWBAN) as being capable of providing instantaneous health status to the user through different sensors. An appealing topic in the latter in coupling of pervasive communications with lightweight portable devices to form pervasive health monitoring so that healthcare personnel can access patient data, review and update them seamlessly. The benefits due to the integration of ubiquitous computing in healthcare include mobility of healthcare, continuity of support in monitoring and treatment, patient status report through wide range of sensors in a home environment, improved patient satisfaction through self-management of the healthcare process, reducing medical errors, remote access provisioning to medical facilities and specialists, etc. [7].

In [18], authors define tele-diagnosis as a service which uses simple applications to facilitate point-to-point asynchronous communication. This service is of high importance in areas that lack expert medical personnel. Usually the remote sites send requests to a medical center and transmit the required data regarding a diagnostic examination, e.g, medical images. The specialists review the data and send back a diagnostic report to the requesting parties.

Tele-consultation, in contrary to tele-diagnosis, is generally defined as synchronous analysis and manipulation of medical data, which requires high QoS [16]. It involves an environment where remote parties (medical experts) can view the same set of medical data at the same time and exchange comments in a real-time manner [18]. In tele-surgery, sometimes termed remote surgery [19], as the name implies, the surgeon is in a remote location and surgery is performed using a robotic system controlled by the surgeon. This service requires robotics in conjunction 
with a high speed communication system as streaming images need to be transferred in real time. The QoS requirements are very strict as the service is sensitive to delay and data loss [16]. Public telecommunication lines, such as Internet, can be used in connection with surgery environments to make tele-surgery a more commonly employed method, although current instances usually use a dedicated line in order to lower the delay and data loss. Tele-surgery would lower the dissimilarities in medical treatments over different domains and also eliminates the risk of infection for doctors [20].

Electronic health record (EHR) is a systematic method of collecting and maintaining patients' data which makes it possible for the health records to be accessible from different hospitals or any other caretaking centers. EHR can contain a wide range of data. Authors in [16] investigate the QoS requirements for EHR and introduce it as a delay tolerant e-Healthcare service. The delay depends on the amount of information to be transmitted but having a reliable transmission with no packet loss is a strict requirement. EHR is accessed at anytime, either it is an emergency or not [16].

Figure 2.4 shows a list of typical medical devices currently being used in the market.

\subsection{Wireless technologies and standards}

There is a wide variety of radio technologies and standards being used in the delivery of wireless e-Healthcare systems. Figure 2.4 summarizes technologies and standards. In [21], authors introduce small cells as improved-capacity systems in comparison to traditional microcells which use IP networks as backhaul rather than conventional cellular network infrastructure. They further investigate the possibility of replacing Wi-Fi technology with femtocell technology.

\section{GSM}

GSM is abbreviated from Global System for Mobile Communications. It is one of the main technologies in second generation $(2 \mathrm{G})$ wireless networks. Some similar technologies based on circuit- 


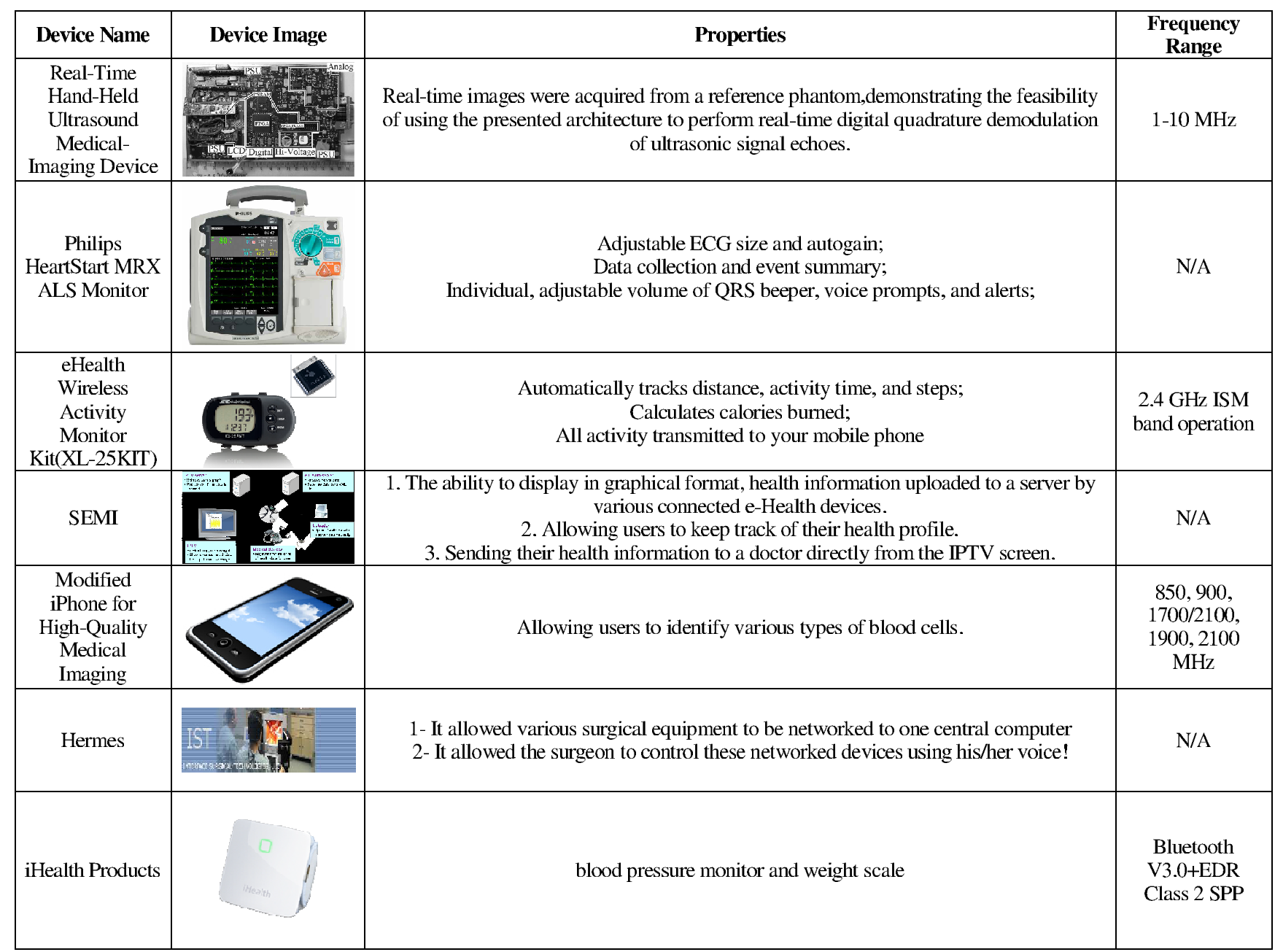

Figure 2.3: Typical e-Healthcare devices. 


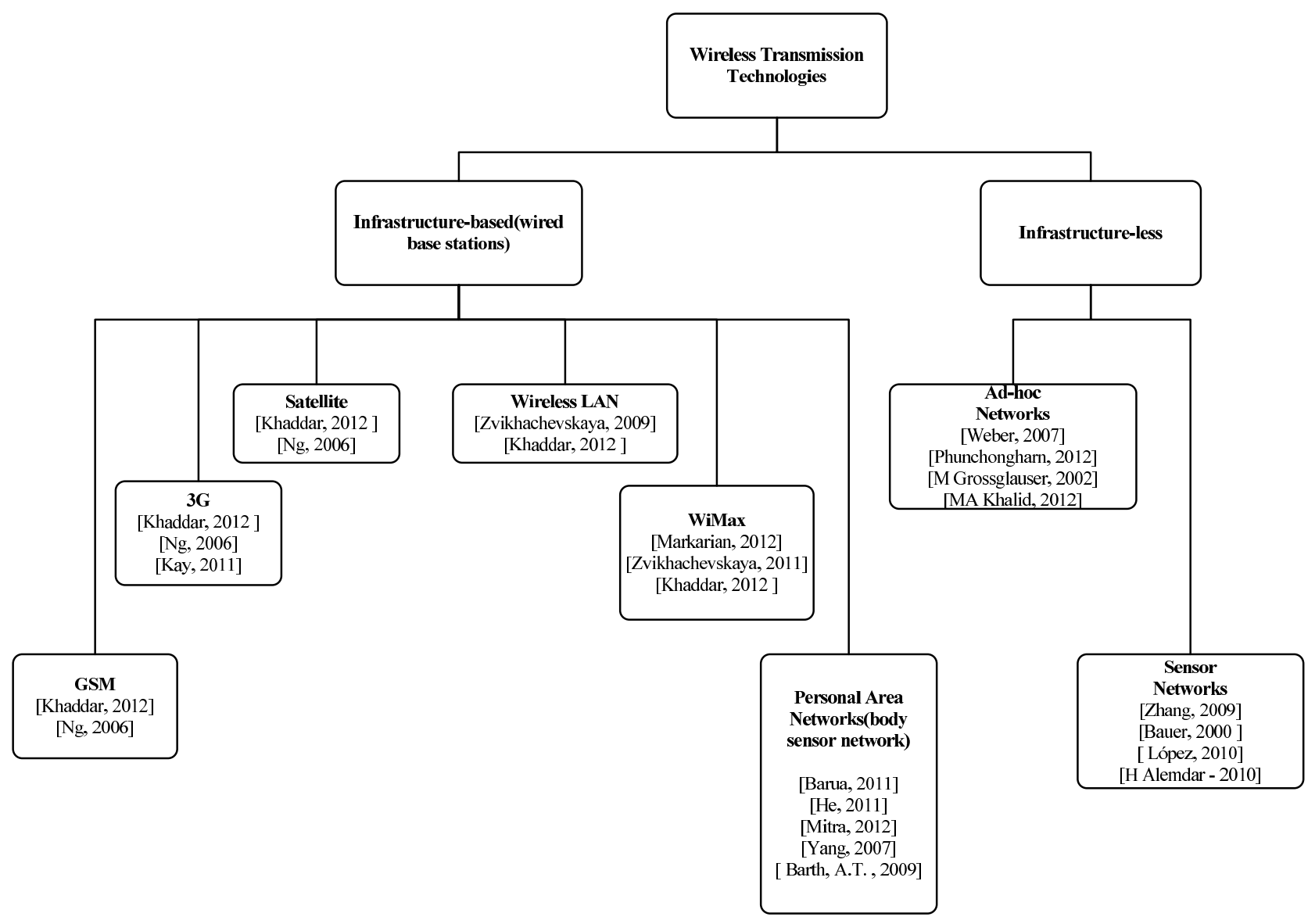

Figure 2.4: Wireless technologies used in wireless e-Healthcare systems 
switched technologies such as Frequency Division Multiple Access (FDMA), Time Division Multiple Access (TDMA), Code Division Multiple Access (CDMA), and Personal Digital Communications (PDC) are also being used in modern e-healthcare industry [7]. The various technologies are used for the transmission of information in healthcare. Most organizations choose technologies for different applications based on throughput, quality, cost and security.

As higher data rate is demanded in e-health, GSM was expanded over time to include data communications, first by circuit switched transport, then packet data transport via GPRS (General Packet Radio Services) and EDGE (Enhanced Data rates for GSM Evolution or EGPRS), which is also named $2.5 \mathrm{G}$ and $2.75 \mathrm{G}$ systems [7]. However, [22] indicates that all existing wireless technologies including GSM will be integrated into a seamless platform by the next-generation mobile communications such as 4G (WiMax or LTE) and beyond-3G (B3G)

\section{G}

Short for third generation, is a term used to represent the 3rd generation of mobile telecommunications technology. It's also called Tri-Band 3G. 3G consists of UMTS system and CDMA2000 system. In UMTS system, the UMTS system, first offered in 2001, standardized by 3GPP, used primarily in Europe, Japan, China (however with a different radio interface) and other regions predominated by GSM 2G system infrastructure. The cell phones are typically UMTS and GSM hybrids. Several radio interfaces are offered, sharing the same infrastructure: The original and most widespread radio interface is called W-CDMA. The TD-SCDMA radio interface was commercialized in 2009 and is only offered in China. The latest UMTS release, HSPA+, can provide peak data rates up to $56 \mathrm{Mbit} / \mathrm{s}$ in the downlink in theory $(28 \mathrm{Mbit} / \mathrm{s}$ in existing services) and 22 Mbit/s in the uplink. the CDMA2000 system, first offered in 2002, standardized by 3GPP2, used especially in North America and South Korea, sharing infrastructure with the IS-95 2G standard. The cell phones are typically CDMA2000 and IS-95 hybrids. The latest release EVDO Rev B offers peak rates of $14.7 \mathrm{Mbit} / \mathrm{s}$ downstream. $3 \mathrm{G}$ is a large-scale wireless network and mobile computing solution, with this technology, healthcare personnel are able to load vital patients' in- 
formation anywhere and anytime within the healthcare network. For instance, $3 \mathrm{G}$ technology is widely used for healthcare personnel to access the hospital database in an ambulance [22].

\section{Satellite}

Though satellite systems are always an alternative option to offer high-speed wireless communications to those underserved and remote regions, because satellite systems cover almost everywhere in the world from the space which means that there are hardly any dead zone [22]. Although satellite system has good coverage and diverse data rates, the operation costs very much [22].

\section{Wireless LAN}

It is short for wireless local area network which links two or more devices using some wireless distribution method (typically spread-spectrum or OFDM radio), and usually providing a connection through an access point to the wider Internet. This gives users the mobility to move around within a local coverage area and still be connected to the network. Most modern WLANs are based on IEEE 802.11 standards, marketed under the Wi-Fi brand name.In a wlan, data can be transferred at a speed of up to $300 \mathrm{Mbits} / \mathrm{s}$ as long as users are within a short distance from the wlan base station or the access point. This distance is generally 30-50 meters indoors and 100-500 meters outdoors [7]. There are mainly 4 standards using in modern e-healthcare which are IEEE802.11a (5Ghz, $54 \mathrm{Mbits} / \mathrm{s})$, IEEE802.11b (2.4GHz, 11Mbits/s), IEEE802.11g (2.4 Ghz, $54 \mathrm{Mbits} / \mathrm{s})$ and IEEE802.11n (2.4GHz and 5GHz, $300 \mathrm{Mbits} / \mathrm{s})$.Wlan is being used very often in lots of situations today as well as for communications between medical devices [23].

\section{WiMAX}

Also known as Worldwide Interoperability for Microwave Access, WiMax is a wireless communications standard designed to provide 30 to 40 megabit-per-second data rates, with the 2011 update providing up to $1 \mathrm{Gbit} / \mathrm{s}$ for fixed stations. In comparison to Wi-Fi, WiMax covers much greater 
distances and provides better QoS than Wi-Fi. Because WiMax has more throughput and less delay, Wi-Fi is being replaced by WiMax to avoid the degradation issues of a mesh Wi-Fi network [7]. Nowadays, WiMAX has been merged with other wireless communications systems, such as Wireless Personal Area Networks (WPANs), Wireless Local Area Networks (WLANs), and cellular systems $(2.5 \mathrm{G}, 3 \mathrm{G}$ and beyond $3 \mathrm{G})$ into a flexible and heterogeneous network, which makes mhealth services more accurate, sufficient and reliable [7] for both fixed and mobile m-health users in a wireless metropolitan area network environment [24].

\section{Personal Area Networks (Body Sensor Network)}

Body sensor network (BSN) are terms used to describe the application of wearable computing devices. This will enable wireless communication between several miniaturized body sensor units (BSU) and a single body central unit (BCU) worn at the human body. With the combination of biosensors and wireless networks, medical personnel are able to pervasively monitor patients' health, behaviors, physiological and contextual parameters [25] at all times [26]. Generally, in BSNs, low power radio is being used to minimize the radiation to human body, reliable and secure wireless links are also being used to transmit sensitive physiological data [25].

\section{Ad-hoc Networks}

A wireless ad hoc network is a decentralized type of wireless network. The network is ad hoc because it does not rely on a preexisting infrastructure, such as routers in wired networks or access points in managed (infrastructure) wireless networks. Instead, each node participates in routing by forwarding data for other nodes, and so the determination of which nodes forward data is made dynamically based on the network connectivity. In addition to the classic routing, ad hoc networks can use flooding for forwarding the data. Since a centralized wired infrastructure is not required in ad-hoc networks, many medical devices can communicate with each other by routing traffic from source to destination through multiple hops [27]. Thus, ad-hoc network is a perfect alternative solution for emergency scenarios when either the existing communication system fails or there are 
no basic infrastructures in rural remote areas [28].

\section{Sensor Networks}

A wireless sensor network (WSN) consists of spatially distributed autonomous sensors to monitor physical or environmental conditions, such as temperature, sound, pressure, etc. and to cooperatively pass their data through the network to a main location. WSN technology is considered as one of the most important research areas in computer networks and e-healthcare application industries [8]. In e-healthcare area, lots of medical applications are developed by working through WSNs in order to improve existing healthcare and monitoring services especially for the elder people and children [8]. Among all benefits, remote monitoring is the main thing for pervasive healthcare system. With remote monitoring, it becomes easier to monitor elder people's conditions to give quick response when emergency happens, also the children will be taken care of in a safer way when they are alone at home. All these jobs can be done without disrupting the patients' normal life.

\subsection{Potential challenges in design and implementation}

\subsubsection{Power allocation}

Conventional noise in wireless communication systems can be overcome by increasing transmit power, but it introduces interference. Therefore, in both centralized and ad hoc wireless networks, if each device only uses the minimum required transmission power, the overall system capacity can be maximized while the interference caused to other devices is minimized [29]. The increased use of low cost Wi-Fi mesh networks makes it possible for some of the eHealthcare applications that require high transmission bandwidth to use Wi-Fi. A Wi-Fi mesh network operates on a multihop basis without the need for high transmission power. However, the delay increases and throughput degrades if there are many hops in between the users and gateway [22]. 
Authors in [30] describe a medium access control (MAC) protocol designed for wireless sensor networks used in e-Healthcare applications, with a goal of minimizing power consumption. The approach focuses on improving collision avoidance and the use of centrally controlled time-slot allocation to sensor nodes. Whereas in [31] for minimizing the power consumption of patient nodes, a simple algorithm is proposed for duty cycling to be implemented in the MAC sublayer of the Zigbee network. The authors conclude that precise duty cycling is the key to optimize network lifetime, considering other factors such as routing method and optimizing router locations.

\subsubsection{Resource management}

In [14], author mentioned that telemedicine and electronic health devices might not have packets to transmit all the time. WBASNs (as an example for WPANs) usually consist of non-identical devices in data rate or packet size. For low-power operations in such networks, IEEE 802.15.4 MAC is a widely used medium access protocol. It supports guaranteed time slot (GTS) allocation which offers scheduled transmissions and therefore saves the energy spent on carrier sensing otherwise. A general discrete time Markov chain is used to model this network. For the operation of IEEE 802.15.4 MAC in beacon enabled mode, this model considers the protocol parameters including idle time, GTS transmission mode, variable back-off window size and low data rate mode.

\subsubsection{QoS support}

Quality of service (QoS) control mechanism is necessary to meet the minimum requirements of a variety of healthcare services which are delivered over networks in conjunction with other commercial traffic (e.g., voice calls, streaming multimedia, and Internet traffic) [16]. QoS requirements for different e-Health services is introduced in Figure 2.5. Thus, a QoS establishment is needed for applications of e-healthcare services to request the required QoS level and adapt to the real QoS offered by the network in reality. With better QoS mechanism, less packet delay and losses are obtained upon network congestion. For example, [32] mentioned that a TCP application may not sustain the same level of QoS as a UDP application because UDP offers one-way guarantees while 


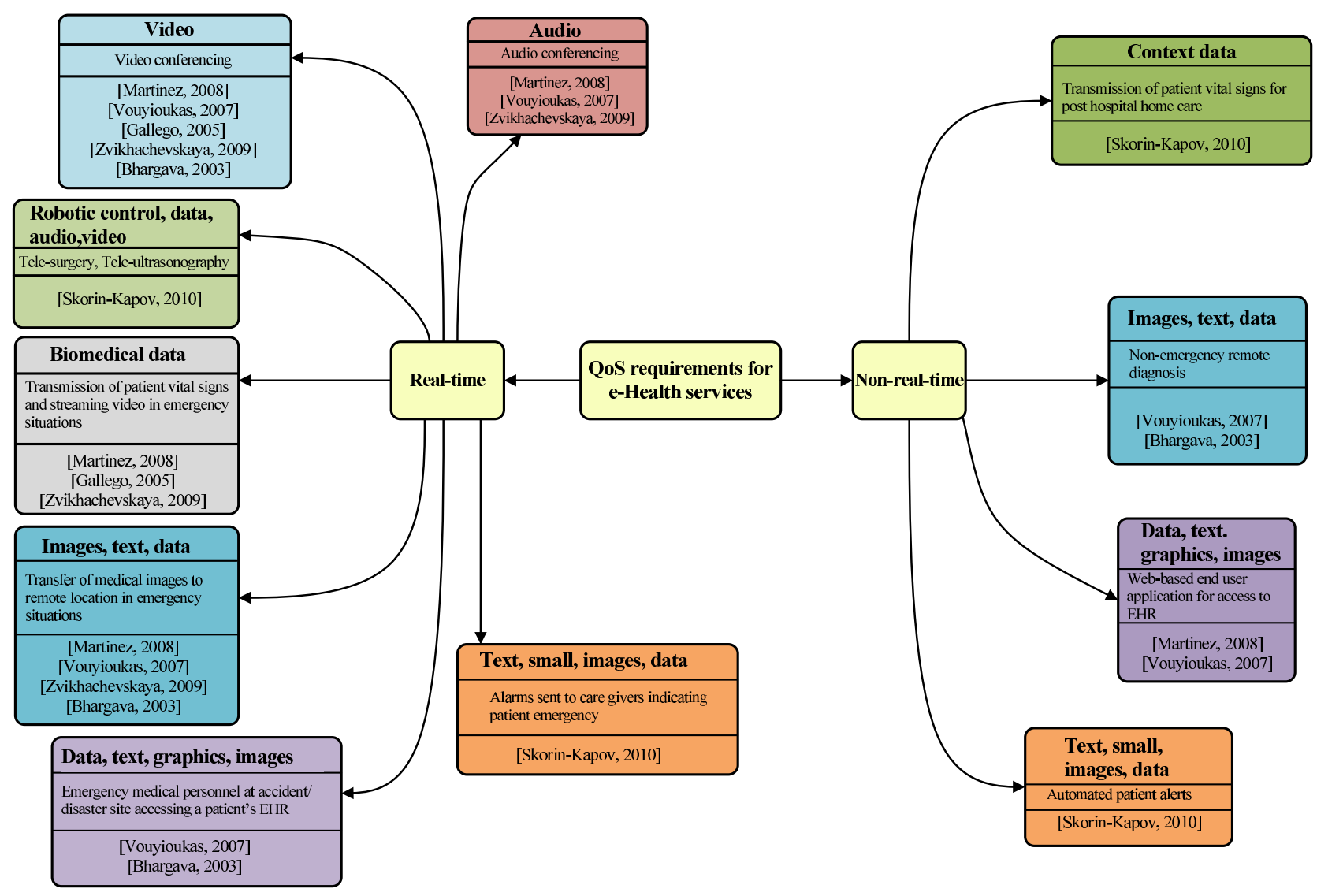

Figure 2.5: QoS requirements for e-Health services

TCP generates a reverse acknowledgement flow in addition to a data flow. QoS has the ability to offer different priority to different applications, users, or data flows when the network capacity is insufficient.

In many services providing QoS, Differentiated Service (DS) and Integrated Service(IS) are two promising ones. Both of them are Better-than-Best-Effort network services offering multiple levels of QoS. DS is a packet-based priority service, offering premium and assured services to meet differentiated requirements of e-healthcare applications. IS is a flow-based reservation service, providing Controlled-Load and Guaranteed services to support delay intolerant services such as real-time service (e.g. tele-consultation services involving transmission of patient physiological parameters in emergency situations [16] [32]).

In e-health or telemedicine services over wireless medical networks, QoS provisioning is 
needed because of the strict requirements and real-time nature of medical applications [33]. Because healthcare networks are supposed to operate 24 hours a day. In medical network, delay is a very important factor, since medical communications should be timely to save critical patients' lives in most cases [23].

Following comes the QoS requirements for e-Healthcare services:

1. Audio: It has become possible to deliver wherever and whenever needed with the high speed wireless communication technology. Audio conferencing is one of the most frequent cases, in which an experienced doctor in the hospital remotely diagnoses patients. In other cases, a guaranteed telephonic communication between the ambulance and the hospital is always necessary. Real-time diagnostic audio applications include the transmission of stethoscope audio, or the transmission of the audio stream with the diagnostic video.

2. Video: Video conferencing combines video and audio, offering the possibility of tele-diagnosis. Sometimes a video communication is required for experienced doctors to know specific situation by watching the inside of the ambulance. Recent Research results show that video is the service with the highest resources demand. Thus, it is more important to guarantee the quality of video services. In [34], A mobile healthcare satellite system is introduced, in which video conferencing data is transmitting at a speed of $384 \mathrm{~kb} / \mathrm{s}$. However, this kind of system costs a lot by using a satellite link.

3. Robotic Control: In robotic tele-ultrasonography systems, high-definition medical images can be delivered with the support of high speed $3 \mathrm{G}$ networks. Delay and loss are intolerant in this case. The acceptable delay time for tele-surgery is generally 330ms [35].

4. Biomedical data: In hospitals, patient's biomedical information is compressed and coded and then transferred to the mobile Intensive Care Unit (ICU) [36] for specialists to do an earlier diagnosis.

5. Medical Images: Medical images transmission is a part of tele-consulation services. Actually, there are no theoretical bandwidth requierments for medical images. The only thing 
that bandwidth matters for medical images is transmitting time. However, some high-quality medical images may require a space of 40 to 50 megabytes, which needs image compression techniques to reduce the bulky image data [34].

6. Data Accessing EHR: Electronic Health Records is invented to include all health data of patients. Mobile access to electronic health records by mobile devices is a part of $m$-health services being implemented in hospitals [34].It belongs to delay-tolerant service, so the required throughput can be low or high, the delay requirement is not very strict for accessing EHR. However no packet loss is allowed in this scenario since patients' EHR data must be complete [35].

7. Alarms Data: Alarms are sent to care givers indicating patient emergency.

8. Context Data: Transmission of patient vital signs for post-hospital home care.

9. Remote Diagnosis data: Some portable medical devices support long-distance tele-diagnosis service by medical staff using GSM or 3G wireless technology. The required throughput is high and delay time must be small for this senario.

10. Patients Alerts Data: Automated patient alerts (e.g., reminder for check up, reminder to take medication

\subsubsection{Emerging technologies}

1. EMI mitigation. In [37] authors describe femtocell base stations as fully user deployable which leads to reduced infrastructure and maintenance costs, but will improve QoS. They introduce interference as an important challenge which arises from mass deployment of femtocells. This directly affects capacity and achievable data rates. The solutions might vary depending on the physical layer technology. Cases of interference include co-channel deployment, adjacent channel deployment, and downlink and uplink interference [38]. Sometimes, many femtocells and their associated macrocell use the same portion of spectrum, 
these can cause interruptions to each other. Authors of [37] introduce different interference management techniques and categorize them as interference cancelation, interference avoidance and distributed interference management.

Interference cancelation includes schemes that reduce or cancel the interference at receiver, after the signal is received. Authors in [29] describe it as a technique that demodulates/decodes the information and uses these information along with channel estimates to cancel the received interference from the received signal. In [37], authors indicate that Interference avoidance is achieved by introducing intelligence to femtocell access points. As these access points face different interference challenges depending on their location and the environment, it is hard to have a centralized control for this matter. Therefore, femtocell access point should be smart enough to cope with different situations and make sure that user receives a good quality service. Distributed interference management schemes are suggested in different recent researches due to impracticality of centralized techniques in larger networks. Femtocells have limited knowledge about the global femtocell network and providing sufficient information to femtocells through the backhaul network causes congestion on the network. Thus, it becomes obvious the importance of distributed schemes. An appealing topic in [39] is about different interference management approaches and names them as cooperation among macrocell base stations (MeNBs) and femtocell base stations (HeNBs), exchange of information in groups of home evolved node Bs (path loss, location, etc.), intelligent spectrum access, etc.

2. Cognitive radio. In [40], authors propose a method for finding an optimal partition of frequency spectrum between microcells and macrocells. To ensure mobile users have connectivity at all times, and to keep the handoff rate at an acceptable level, high-mobile users (those who move with a higher speed than a reference velocity) should have handoffs at macrocell boundaries and accordingly low-mobile users should undergo handoffs at microcell boundaries. 
As it is known, femtocell has been a popular topic in the field of wireless technology and deployed in many medical environment. Meanwhile, cognitive radio based on distributed spectrum sensing can be merged with femtocell for interference mitigation. One of them is called cognitive femtocell with cognitive functions, which makes femtocell network intelligent enough to offer better indoor coverage and capacity [41]. A cognitive femtocell can utilize the spectrum efficiently with the property of opportunistic spectrum access property, as well as avoiding interference by its ability to recognize an interference signature which can be used for femtocell to select certain channels that are not having interference problem [41].

3. Physical layer. The MAC layer is a sub-layer between the physical layer and network layer, handling the access to the communication channel and offering a reliable link between two MAC entities. The design of a MAC layer protocol may impact some key factors in transmission of medical application data such as throughput, power consumption, transmission delay and quality of service [42]. Power consumption has been a highly focus of attention in the most recent research. More and more e-healthcare applications such as vital signs monitoring using wireless sensor network technologies rely on MAC protocols such as Bluetooth and 802.11. But, one disadvantage is that they are mostly enormous and power hungry. To solve this problem, in [30], a novel energy-efficient MAC protocol designed specifically for wireless body area networks (WBASN) focused towards pervasive healthcare applications is introduced. The proposed MAC protocol aims to lower the power consumption from the sources like idle listening, power outspending and collision by focusing on collision avoidance and using centrally controlled time slotting for sensor nodes as well as cross-layer optimization, performing upper layer functions. 


\subsection{Chapter Summary}

The improvements in computing and communication systems have had great influence in different aspect of our lives. One of the most important fields relates to healthcare. Trustworthiness of sensors, electronic devices, networks and wireless communications have reached to an extent that they can replace traditional legacy systems. Therefore, the term e-Healthcare is introduced. Regardless of all tremendous advances in this field, there still remains unexplored aspects of it especially considering the challenges. In this chapter, we discussed different elements of e-Healthcare, introduced wireless transmission technologies and standards that are used in e-Healthcare, presented the QoS requirements of each service, and discussed the potential challenges in power allocation, scheduling, meeting the QoS needs, and confronting the electromagnetic interference. Pervasive and continuous health monitoring is one of the most widely utilized services all over the world. It specifically provides the elderly and chronically ill patients with continuous and seamless health monitoring, benefiting both them and the caretakers.

In this chapter, power allocation, QoS support, EMI mitigation and cognitive radio in this chapter are partially covered. The topology of a single floor in a hospital and wireless network environment are described in the first section of next chapter. In this environment, we formulate a multi-objective optimization problem for joint admission control, green power allocation in cognitive radio system, using weighted sum method in the second section. For the last section, we propose an modified branch and bound algorithm to solve our problem using OPTI Toolbox in order to obtain the final solution. 


\section{Chapter 3}

\section{Communication Network Model in Hospital}

\subsection{Communication Network in Hospital}

Figure 3.1 shows a single floor of a hospital, where there are three areas and two hallways making a department within a hospital. There are patients, doctors, nurses, specialists and supporting staff working in this floor. Specialists and supporting staff are responsible for operating all medical equipments [43].

On top right corner of the figure, there are some specialists, a few physicians and nurses working in a room named central monitoring room. This room provides quality patient care including real-time monitoring and clinical decision support tools. It provides nurses with a clear view of patient's status. For the doctors, it enables access to timely patient monitoring information where and when it's needed. It can communicate with hospital information systems (HIS). On left side of the central monitoring room, people can go in and out through the main door of this floor. One more step to the left, there are one small nursing unit and one large nursing unit. Patients are taken care of in this area. Inside these units, there are a few private patient rooms with individual bathrooms and showers to improve comfort and reduce infection. There is also a quiet room for rounds and private exchange of patient information, teaching room for residents, private consult room with computer, spacious main nurses' station, physician station and staff lounge to enhance 


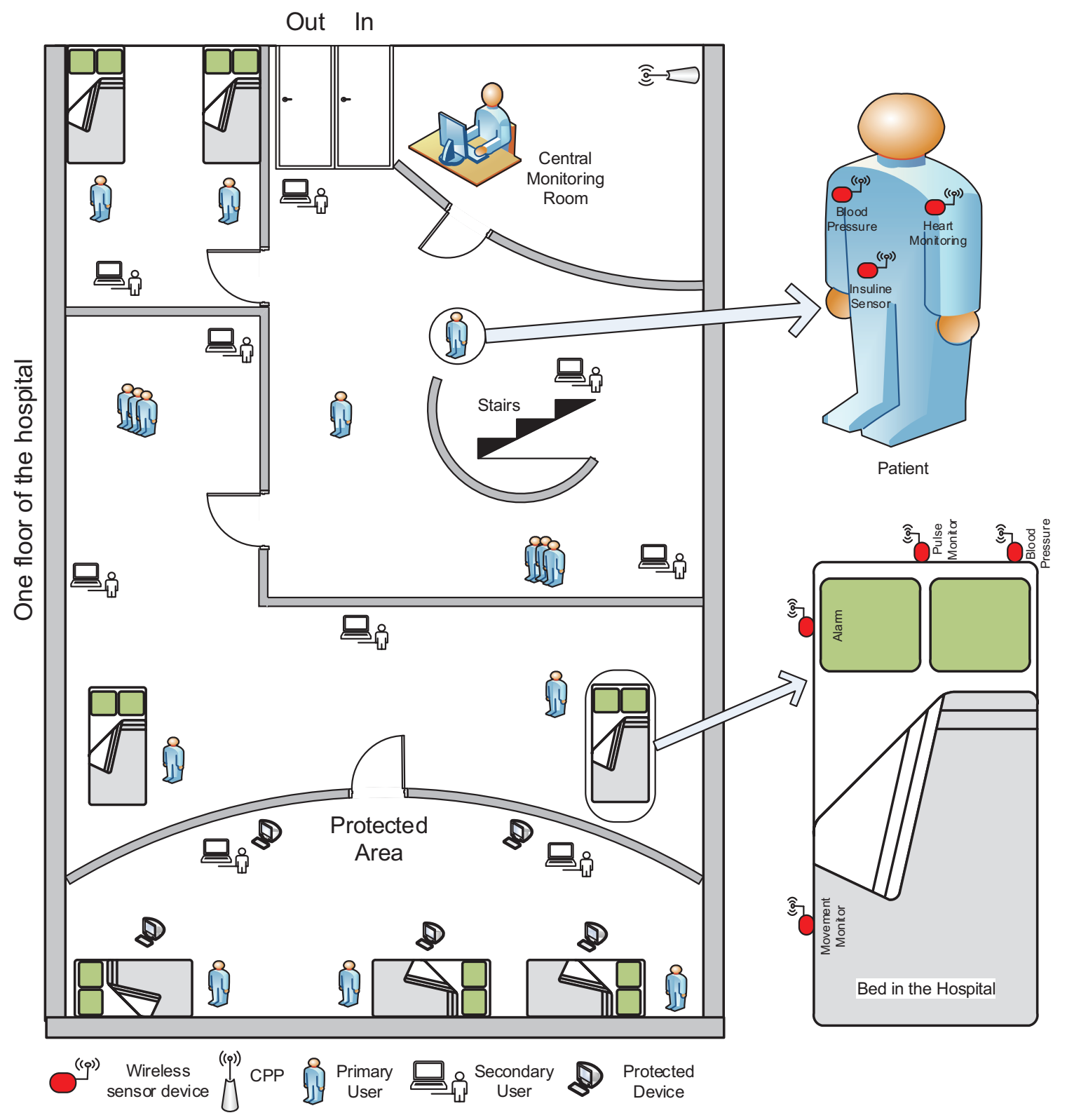

Figure 3.1: Network model

communication and efficiency in patient care.

Surrounded by nursing unit and central monitoring room, there is a large hall for people walking around and to talk with each other. In the centre of the hall, there is one stairway to other floors 
in the hospital. At the very bottom of the floor, we have one protected area. Setting protected area is a method for patients' protection. Protected areas are classified as body or cardiac protected areas. The procedures carried out to classify these areas are as follows: Within the body protected areas, procedures are carried out where a patient is connected to an electromedical equipment which reduces the impedance of the skin by either electrode paste, for example.

Besides human beings in this hospital, large number of medical devices are deployed in this floor as well. All medical devices we deploy in this floor are mainly used for gathering patients' information, such as patient health record, high-definition medical images and real-time video. These data can be accessed by not only specialists, doctors and nurses, but the medical equipments and health care applications or softwares as well. All the medical devices such as computers, realtime surveillance system, real time telemedicine system are connected to the cognitive radiobased communication network. Mobile devices, such as personal laptops, iPads and some other smart tablets can also be connected to this network using different channels. For all wireless devices in this cognitive radio network, the communication is through multiple channels in an unlicensed spectrum band. In this network, the central processing point (CPP) is the controller, other devices in this floor are all cognitive radio clients. The CPP located in the centre on the ceilling of this floor can transmit or receive data from all cognitive radio clients and control the entire operations.

As illustrated in Fig. 3.1, there are three types of users in the hospital.

- Protected users are passive medical devices (e.g., incubators and ECG monitors) located in protected area at the bottom of the hospital topology, do not transit any data, but they are very sensitive to EMI (electro-magnetic interference) which should be protected. For each primary user and his bed, there are several wireless sensors attached around to transmit electronic health information about the patient wirelessly.

- Primary users are active medical devices (e.g., telemetry monitors, insuline sensor and pulse monitor), and they can transmit wireless signals, intended for therapeutic use. 
- Secondary users are another kind of active medical devices (e.g., used for hospital information system and testing or calibration devices), can transmit data opportunistically, intended for testing, calibration, learning and research [44] [45].

Transmissions from secondary users can interfere transmissions from primary and protected users, thus, secondary users only transmit wirelessly when they do no harmful interference to the other two types of users.

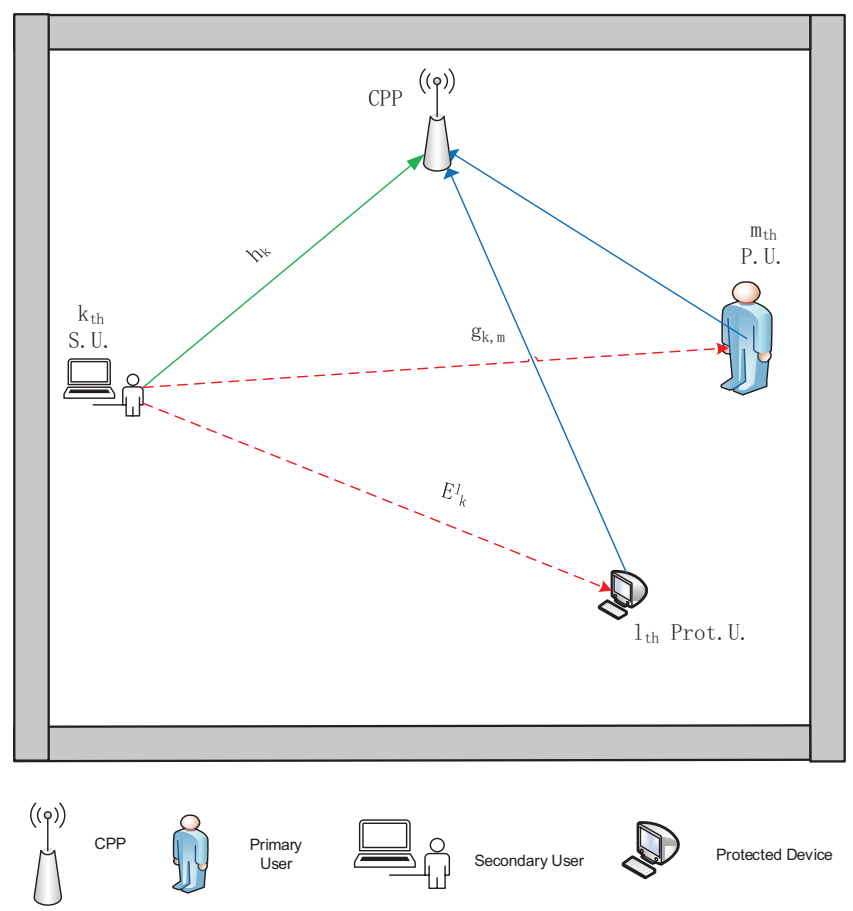

Figure 3.2: Network interference model

We consider a small cell wireless network in one floor in a hospital, consisting of one CPP (central processing point) located in the centre of this floor, $K$ secondary users, $M$ primary users and $L$ protected users in the protected area. $\mathcal{K}$ denotes a set of secondary users, $\mathcal{M}$ denotes a set of primary users, $\mathcal{L}$ denote a set of protected users. We consider uplink communication as shown in Figure 3.2. Each user employs only a single antenna for commmunication, all users are transmitting signal on the same frequency band. $K$ users are on the secondary network and $M$ users on primary network. When secondary users are active, each secondary user transmits signal in its own channel, which means $K$ secondary users are transmitting signals through $K$ channels, 
and hence there are no interference among secondary users. However, when $m_{t h}$ primary user becomes active, it can transmit signal over any channel of those being used by secondary users from $1_{s t}$ to $k_{t h}$ secondary user. Thus, this will cause interference between $m_{t h}$ primary user and surrounding secondary users who are transmitting at the same time. As Figure 3.2 indicates, when $k_{t h}$ secondary user is transmitting signals to $\mathrm{CPP}$, its source power interferes other types of users ( $m_{t h}$ primary user and $l_{t h}$ protected user) through the channel $g_{k, m}$ and $E_{k}^{l}$. We want to admit a

Table 3.1: Key Notations

\begin{tabular}{|c|c|}
\hline Symbol & Meaning \\
\hline$\phi$ & Set of selected secondary users for communication \\
\hline$|\phi|$ & Number of selected secondary users for communication \\
\hline $\mathcal{K}$ & Set of secondary users \\
\hline$K$ & $K=|\mathcal{K}|$, number of secondary users \\
\hline $\mathcal{M}$ & Set of primary users \\
\hline$M$ & $M=|\mathcal{M}|$, Number of primary users \\
\hline $\mathcal{L}$ & Set of protected users \\
\hline$L$ & $L=|\mathcal{L}|$, Number of protected users \\
\hline$P_{s}$ & Maximum of source power when all users are transmitting signals \\
\hline$p_{k}$ & the source power in $k_{t h}$ user band transmitted by $k_{t h}$ secondary user \\
\hline $\mathbf{p}$ & $\mathbf{p}$ is a vector, $\mathbf{p}=\left[p_{1}, p_{2}, p_{3} \ldots \ldots p_{K}\right]$ \\
\hline$x_{k}$ & $x_{k}$ is variable,$x_{k}=0$ or 1 \\
\hline $\mathbf{x}$ & $\mathbf{x}$ is a vector, $\mathbf{x}=\left[x_{1}, x_{2}, x_{3} \ldots \ldots x_{K}\right]$ \\
\hline$X$ & a constant in grams/Watt \\
\hline$e_{k}$ & $e_{k}=X p_{k}$ \\
\hline$E^{\max }$ & $E^{\max }=X P_{s}$ \\
\hline$W$ & channel bandwidth \\
\hline$c_{k}$ & transmission rate of secondary user $k$ \\
\hline$C^{\max }$ & maximum of $c_{k}$ \\
\hline$C_{t h}$ & minimum rate requirement for secondary users \\
\hline$h_{k}$ & the channel from $k_{t h}$ secondary user to CPP \\
\hline$h_{k}^{\max }$ & $h_{k}^{\max }$ is the best channel gain from $k_{t h}$ secondary user to CPP. \\
\hline$N_{0}$ & noise power \\
\hline$g_{k, m}$ & the channel from $k_{t h}$ secondary user to $m_{t h}$ primary user \\
\hline$I_{m}$ & Maximum of allowed interference at the $m_{t h}$ primary user \\
\hline$w_{1}, w_{2}, w_{3}$ & Weights, $w_{1}+w_{2}+w_{3}=1$ \\
\hline$E_{k}^{l}$ & the electric field from secondary user $k$ to a protected device $l$ \\
\hline$d_{k}^{l}$ & the distance between the protected user $l$ and the secondary user $k$ \\
\hline$Z$ & constant from the free-space impedance of protected device $l$ \\
\hline
\end{tabular}

certain number of secondary users that are allowed to transmit wireless signals, that is to control 
the admission. Therefore, we let $\phi$ denote a set of selected users from the secondary network, and $|\phi|$ denotes number of selected users $(|\phi| \epsilon 1,2,3 \ldots K)$. We denote by $p_{k}$, the source power in $k_{t h}$ secondary user band, $P_{s}$, maximum of source power. We define that $\mathbf{p}$ is a vector, $\mathbf{p}=\left[p_{1}, p_{2}\right.$, $\left.p_{3} \ldots . . p_{K}\right] . x_{k}$ is variable, $x_{k}=1$ when $k_{t h}$ user is transmitting, otherwise it's 0 . We also define that $\mathbf{x}$ is a vector, $\mathbf{x}=\left[x_{1}, x_{2}, x_{3} \ldots \ldots x_{K}\right] . W$ is channel bandwidth. As data traffic rates are increasing and radio transmission is more advanced these days, wireless networks consume a larger amount of energy and contribute a increasing amount of $\mathrm{CO}_{2}$ emissions. Thus, we also research energy consumption and energy efficiency of wireless networks, especially we focus on $\mathrm{CO}_{2}$ emissions of the mobile network infrastructure in hospital environment. $X$ is a constant in grams/Watt related to $\mathrm{CO}_{2}$ emissions. $e_{k}$ and $E^{\max }$ are introduced in next section. $c_{k}$ is transmission rate of secondary user $k . C^{\max }$ is maximum of $c_{k}$. $C_{t h}$ is minimum rate requirement. We denote by $h_{k}$, the channel from $k_{t h}$ secondary user to destination. $h_{k}^{\max }$ is the best channel gain among all secondary users. We denote by $g_{k, m}$, the cannel from $k_{t h}$ secondary users to $m_{t h}$ primary user, $I_{m}$, maximum of allowable interference at the $m_{t h}$ primary user. $w$ denotes weight. $N_{0}$ is noise power in each channel. $E_{k}^{l}$ is the electric field from secondary user $k$ to a protected device $l . d_{k}^{l}$ is the distance between the protected user $l$ and the secondary user $k . Z$ is constant from the free-space impedance of protected device $l$.

\subsection{Weighted Resource Maximization Problem}

Based on the previous discussion, in this section, we formulate a multi-objective optimization problem for joint admission control and green power allocation in cognitive radio network, using weighted sum method. Also, we transform our multi-objective formula into a min-max formula mathematically for convenient calculation.

Our first objective is to maximize the number of selected users, that is,

$$
\max _{\mathbf{p}, \phi} \frac{|\phi|}{|K|}
$$


Here $p_{k}$ is the source power of $k_{t h}$ secondary user in $k_{t h}$ user band. $\mathbf{p}$ is a vector, $\mathbf{p}=\left[p_{1}, p_{2}\right.$, $\left.p_{3} \ldots . . . p_{k}\right] .|\phi|$ is number of selected users (or admitted users). $K$ is number of secondary users that can potencially communicate to CPP. We devide the number of admitted users by the total number of secondary users normalization in order to maximize the percentage of admitted users in the wireless network. As we want to deal with the minimization problem for the final expression, this formula is expressed in another form as:

$$
\min _{\mathbf{p}, \phi}\left(1-\frac{|\phi|}{|K|}\right)
$$

We define a binary variable $x_{k}$ :

$$
x_{k}= \begin{cases}1, & \text { if } k_{t h} \text { secondary user is admitted } \\ 0, & \text { otherwise }\end{cases}
$$

Also, we define another vector $\mathbf{x}=\left[x_{1}, x_{2}, x_{3} \ldots \ldots x_{K}\right]$. Then, the total number of selected users can be written as :

$$
\sum_{k=1}^{|K|} x_{k}=|\phi|
$$

Thus, our first formula then becomes :

$$
\min \left(1-\frac{\sum_{k=1}^{|K|} x_{k}}{|K|}\right)
$$

Our secondary objective is to minimize the $\mathrm{CO}_{2}$ emissions. The $\mathrm{CO}_{2}$ emissions are calculated in grams/Watts. Thus, we denote by $X$, a constant in grams/Watt. We devide source $\mathrm{CO}_{2}$ emissions, which are generated by actual transmissions by the number of secondary users, by the maxmium amount of source $\mathrm{CO}_{2}$ emissions. Then our secondary objective can be written as:

$$
\min _{\mathbf{p}, \mathbf{x}} \frac{\sum_{k=1}^{|K|} x_{k} e_{k}}{E^{\max }}
$$


where $e_{k}=X p_{k}, E^{\max }=X P_{s}$. And $p_{k}$ is the source power in $k_{t h}$ user band, $P_{s}$ is maximum of all the source power. Our goal is expressed by dividing the total amount of $\mathrm{CO}_{2}$ emissions originating from all the selected users by the maximum total power transmitted in the system.

Our third objective is to maximize the data rate of each user such that each secondary user's data rate satisfies its minimum requirement. We first define two variables: $c_{k}$ is transmission rate of secondary user $k, C^{\max }$ is maximum of it for all k. According to Shannon theory [46], it is calculated as follows:

$$
\begin{array}{r}
c_{k}=W \log \left(1+\frac{p_{k} h_{k}}{N_{0}}\right) \\
C^{\text {max }}=W \log \left(1+\frac{P_{s} h_{k}^{\text {max }}}{N_{0}}\right)
\end{array}
$$

where $W$ is channel bandwidth, $h_{k}^{\max }$ is the best channel gain among $|\phi|$ secondary users. Same as our first objective, we can write our third expression mathematically as:

$$
\min _{\mathbf{p}, \mathbf{x}}\left(1-\frac{\sum_{k=1}^{|K|} x_{k} c_{k}}{C^{\max }}\right)
$$

We have five constraints in the final formulation. The condition $\mathrm{C} 1$ controls the total transmission power in the uplink to be less than the maximum of source power for all secondary users. The condition $\mathrm{C} 2$ ensures that the interference between secondary user and primary user is always in the allowable range, this is very important for all electronic and medical devices working properly in a hospital using the same frequency spectrum. Here we assume that all $K$ channels can be used by primary user $m$, and the total interference from all the $K$ channels for primary user $m$ is kept below a threshold $I_{m}$. The condition $\mathrm{C} 3$ ensures that every transmission of secondary user $k$ has a good quality. The fourth condition $\mathrm{C} 4$ is another interference constraint, which is to limit the transmitting power of each user to reduce as much EMI as possible to the protected user [44]. For a protected user $l, E_{k}^{l}$ is the electric field from secondary user $k$ to a protected device $l . d_{k}^{l}$ is the 
distance between the protected user $l$ and the secondary user $k . Z$ is constant from the free-space impedance of protected device $l[44]$.

To summarize it, our formulation is a multi-objective convex mixed integer non-linear programming (MINLP) problem. We use weighted sum method for this to combine the multiple objectives in the optimization problem with $w_{1}, w_{2}, w_{3}$ weights, respectively for call admission control, for green power allocation and maximizing throughput to secondary users. Our final objective expression is expressed as follows:

$$
\begin{aligned}
& \min _{\mathbf{p}, \mathbf{x}} w_{1}\left(1-\frac{\sum_{k=1}^{K} x_{k}}{K}\right)+w_{2} \frac{\sum_{k=1}^{K} x_{k} e_{k}}{E^{\max }}+w_{3}\left(1-\frac{\sum_{k=1}^{K} x_{k} c_{k}}{C^{\max }}\right) \\
& \text { subject to: } \\
& C 1: \sum_{k=1}^{K} p_{k} \leqslant P_{s} \\
& C 2: \sum_{k=1}^{K} p_{k} g_{k, m} \leqslant I_{m}, \forall m \\
& C 3: c_{k} \geq x_{k} C_{t h}, \forall k \\
& C 4: 0 \leq p_{k} \leq x_{k}\left(\frac{E_{k}^{l} d_{k}^{l}}{Z}\right)^{2} \\
& C 5: w_{1}+w_{2}+w_{3}=1
\end{aligned}
$$

We define a variable fval:

$f v a l=$ the objective function value $=w_{1}\left(1-\frac{\sum_{k=1}^{K} x_{k}}{K}\right)+w_{2} \frac{\sum_{k=1}^{K} x_{k} e_{k}}{E^{\text {max }}}+w_{3}\left(1-\frac{\sum_{k=1}^{K} x_{k} c_{k}}{C^{\text {max }}}\right)$

\subsection{Mixed Integer Non-linear Program}

In this section, we prove our problem given in (3.11) is a convex mixed integer non-linear programming problem (convex MINLP). MINLP is abbreviation of mixed integer nonlinear programming. It refers to a mathematical programming problem, in which variables can be continuous or discrete, containing integer and non-integer variables, and nonlinearity for the objective function and constraints. MINLP problem is an important category of optimization problems, which can be applied 
in various real-world problems. For example, there are a lot of applications of MINLP applied in financial, engineering design problems, chemical engineering, process operations research and management [47].

In general, a MINLP problem is :

$$
\begin{array}{r}
\text { minimize: } f(x, y) \\
\text { subject to: } g(x, y) \leq 0 \\
x \in X, y \in Y \\
X=\left\{x \mid x \in R^{n}, x^{L} \leq x \leq x^{U}, B x \leq b\right\} \\
Y=\{y \mid y \in\{0,1\}, A y \leq a\}
\end{array}
$$

The fnuticon $f(x, y)$ is a nonlinear objective function and $g(x, y)$ is a nonlinear constraint function. $x$ and $y$ are the variables bounded over $X$ and $Y$, where $y$ is required to be integer valued. $L$ is lower bound, $U$ is upper bound. $A, B, a, b$ are all real numbers.

In MINLP problems, the objective function and the constraints always include mixed integer problems (MIP), non-linear problems (NLP) and sometimes non-convex problems. Thus, MINLP problems are generally difficult to solve. However, many approaches have been proposed and used to solve MINLP problems. For convex MINLP problems, there are many methods including Branch and Bound (BB), Outer-Approximation (OA), Extended Cutting Plane Method (ECP), and Generalized Disjunctive Programming (GDP) to solve convex MINLP problems [47].

In our MINLP problem, $\mathbf{x}$ is integer and $\mathbf{p}$ is non-integer based on our variables definitions:

$$
x_{k}= \begin{cases}1, & \text { if } k_{t h} \text { secondary user is admitted } \\ 0, & \text { otherwise }\end{cases}
$$

Besides, our objective function includes two non-linear parts which easily makes our problem to 
be a MINLP problem.

In the next step, we prove our objective function to be a convex function using an example. We consider a simple scenario with two secondary users in our system. It is hard to prove the function is convex or not when the number of variables is greater than two, thus we only consider this simple case with two users as the example. In this scenario, $K=2, x_{1}=x_{2}=1$, we use two symbols $a$ and $b$ to denote the source power of user 1 and user 2 , respectively, $p_{1}=a, p_{2}=b$. Since both users are transmitting, the value of our first objective is zero. We assume that power control and throughput guarantee are of equal importance, then we have $w_{2}=w_{3}=1 / 2$. We assume that these two secondary users are having equal wireless channel gains, so we have $h_{1}=h_{2}=1 / 2$. We also use values of constants from Table 4.1 into our function in order to do more convenient calculation. For example, $\mathrm{X}=655, E_{\max }=0.655$ and $N_{0}=10^{-8}$.

Thus, our objective funtion in (3.11) becomes:

$$
\begin{array}{r}
f(a, b)=0+\frac{1}{2}\left(\frac{655 a+655 b}{0.655}\right)+\frac{1}{2}\left[1-\frac{10^{4} \log _{2}^{\left(1+\frac{0.5 a}{10^{-8}}\right)}+10^{4} \log _{2}^{\left(1+\frac{0.5 b}{10^{-8}}\right)}}{10^{4} \log _{2}^{10^{5}}}\right] \\
=\frac{1}{2}+500 a+500 b-\frac{1}{2} \log _{10^{5}}^{\frac{a b}{4 \times 10^{-16}}}
\end{array}
$$

We use Hessian matrix to determine whether our function is convex or non-convex by calculating the determinant value of Hessian matrix. The Hessian matrix or Hessian is a square matrix of second-order partial derivatives of a function. It describes the local curvature of a function of many variables. We apply Hessian matrix for our scenario: Given the real-valued function $f(a, b)$ if all second partial derivatives of $f$ exist and are continuous over the domain of the function, then the Hessian matrix of $f$ is

$$
H(f)=\left|\begin{array}{cc}
\frac{\partial^{2} f}{\partial a^{2}} & \frac{\partial^{2} f}{\partial a \partial b} \\
\frac{\partial^{2} f}{\partial b \partial a} & \frac{\partial^{2} f}{\partial b^{2}}
\end{array}\right|
$$


Next, we calculate each partial second derivative of the function.

$$
\begin{gathered}
\frac{\partial^{2} f}{\partial a^{2}}=\frac{\partial f}{\partial a}\left[0+0+500-\frac{1}{2} \frac{4 \times 10^{-16}}{a b} \log _{10^{5}}^{e} \frac{b}{4 \times 10^{-16}}\right] \\
=\frac{\partial f}{\partial a}\left[500-\frac{\log _{10^{5}} e}{2 a}\right] \\
=\frac{\log _{10^{5}} e}{2 a^{2}} \\
\frac{\partial^{2} f}{\partial b^{2}}=\frac{\partial f}{\partial b}\left[0+0+500-\frac{1}{2} \frac{4 \times 10^{-16}}{a b} \log _{10^{5}}^{e} \frac{a}{4 \times 10^{-16}}\right] \\
=\frac{\partial f}{\partial b}\left[500-\frac{\log _{10^{5}} e}{2 b}\right] \\
\frac{\partial^{2} f}{\partial b \partial a}=\frac{\partial f}{\partial b}\left[500-\frac{\log _{10^{5}} e}{2 a}\right]=0 \\
\frac{\log _{10^{5}} e}{2 b^{2}} \\
=\frac{\partial f}{\partial a}\left[500-\frac{\log _{10^{5}} e}{2 b}\right]=0
\end{gathered}
$$

Thus, our Hessian matrix can be calculated as:

$$
\begin{gathered}
H(f)=\left|\begin{array}{cc}
\frac{\partial^{2} f}{\partial a^{2}} & \frac{\partial^{2} f}{\partial a \partial b} \\
\frac{\partial^{2} f}{\partial b \partial a} & \frac{\partial^{2} f}{\partial b^{2}}
\end{array}\right| \\
H(f)=\left|\begin{array}{cc}
\frac{\log _{10^{5}} e}{2 a^{2}} & 0 \\
0 & \frac{\log _{105} e}{2 a^{2}}
\end{array}\right|
\end{gathered}
$$




$$
\begin{array}{r}
H(f)=\left(\frac{\log _{10^{5}} e}{2 a^{2}}\right)\left(\frac{\log _{10^{5}} e}{2 a^{2}}\right)-0^{2} \\
=\frac{\left(\log _{10^{5}} e\right)^{2}}{2 a^{2} b^{2}}
\end{array}
$$

Based on the theorem about second derivative of convex or concave function, which is:

Let $f$ be a real function which is twice differentiable on the open interval $(a . . b)$. Then:

$f$ is convex on $(a . . b)$ if its second derivative $D^{2} f \geq 0$ on $(a . . b)$

$f$ is concave on $(a . . b)$ if its second derivative $D^{2} f \leq 0$ on $(a . . b)$.

This can be proved for general case. In our case, both $a$ and $b$ are power of secondary users, which are positive numbers. According to the result of the Hessian Matrix, the value of it is positive. Therefore, we can prove that our problem given in (3.11) is convex.

\subsection{Proposed Branch-and-Bound Algorithm}

In this section, we propose to use the branch and bound algorithm to solve our formulated program. In order to find the solution to the optimization problem, we use OPTimizition Interface Toolbox (OPTI Toolbox). It is a MATLAB toolbox for constructing and solving linear, nonlinear, continuous and discrete optimization problems. With OPTI Toolbox, we can construct optimization problems and solve them using a range of supplied solvers. OPTI Toolbox is able to automatically detect the problem type being solved and uses the best solver available for the problem, using powerful open source solvers such as IPOPT, SCIP, NOMAD and others. It can also perform solver benchmarking, plot optimization contours and validate the solution.

In our case, we use OPTI Toolbox to solve our optimazition problem which is a convex MINLP. Referring to the problem example on OPTI Toolbox website, the goal of our MINLP is to minimize the objective function by selecting values of vector $\mathbf{x}$ and $\mathbf{p}$ that also satisfies all constraints. The values of $\mathbf{x}$ take discrete (integer) values only. We have opted to split the constraints into linear and nonlinear types. While the MINLP solver will call them all in a single nonlinear callback, OPTI will recognise two constraints as being linear, and pass this linear information to the solver 
to use. We used the same commands and formats for each part of programming such as objective, linear constraints, bounds, and initial guess, etc. In Figure 3.3, the flow chart shows how we use OPTI Toolbox to solve the MINLP problem. OPTI uses BONMIN (the default MINLP solver), which stands for Basic Open Source Nonlinear Mixed Integer Programming. It solves smooth, twice differentiable, mixed integer nonlinear programs. BONMIN uses IPOPT for solving relaxed problems as the mixed integer solver. It contains a number of algorithms for convex MINLPs [48]. For solving MINLP, we choose branch and bound (BB) approach, which is an efficient way to solve MINLP.

Branch-and-bound is a classical algorithm [49] for solving MINLP. One ingredient is a candidate solution, i.e., a point feasible for MINLP. It is updated as soon as a feasible point with a lower objective value is encountered. As such, the candidate solutions yield a decreasing sequence of upper bounds on the optimal objective value of MINLP. Note that a candidate solution might not yet be known at the beginning of the algorithm. Also, the algorithm maintains a set of restrictions of MINLP, for which some of the lower (respectively, upper) bounds on integer variables are increased (respectively, decreased) from their original value. The set of restrictions is chosen so that if there exists a better candidate than the current one, it is feasible for at least one of the restrictions in the set. Note that the optimal objective function value of the continuous relaxation of a restriction is a lower bound on all integer-feasible points of this restrictions. At the beginning, the set of restrictions is initialized to contain the original formulation MINLP.

Branch-and-bound algorithms are used extensively for mixed-integer linear programming models. The basic algorithm is applicable to models with nonlinear functions where a nonlinear solver needs to evaluate the relaxed sub-problems. In [50], a standard branch-and-bound algorithm for MINLP problems with integer variables is introduced which is discussed as Algorithm 1. In this algorithm, $L B$ is lower bound of the function value, $U B$ is the upper bound of the function value, obj is the function value in the particular node $j$, and $x_{k}$ is non-integer variable in the function. We apply this algorithm for our own problem where there are different $L B, U B$, and variables. In our problem, the function value is between 0 and 1 . Also, our non-integer variable should be power 


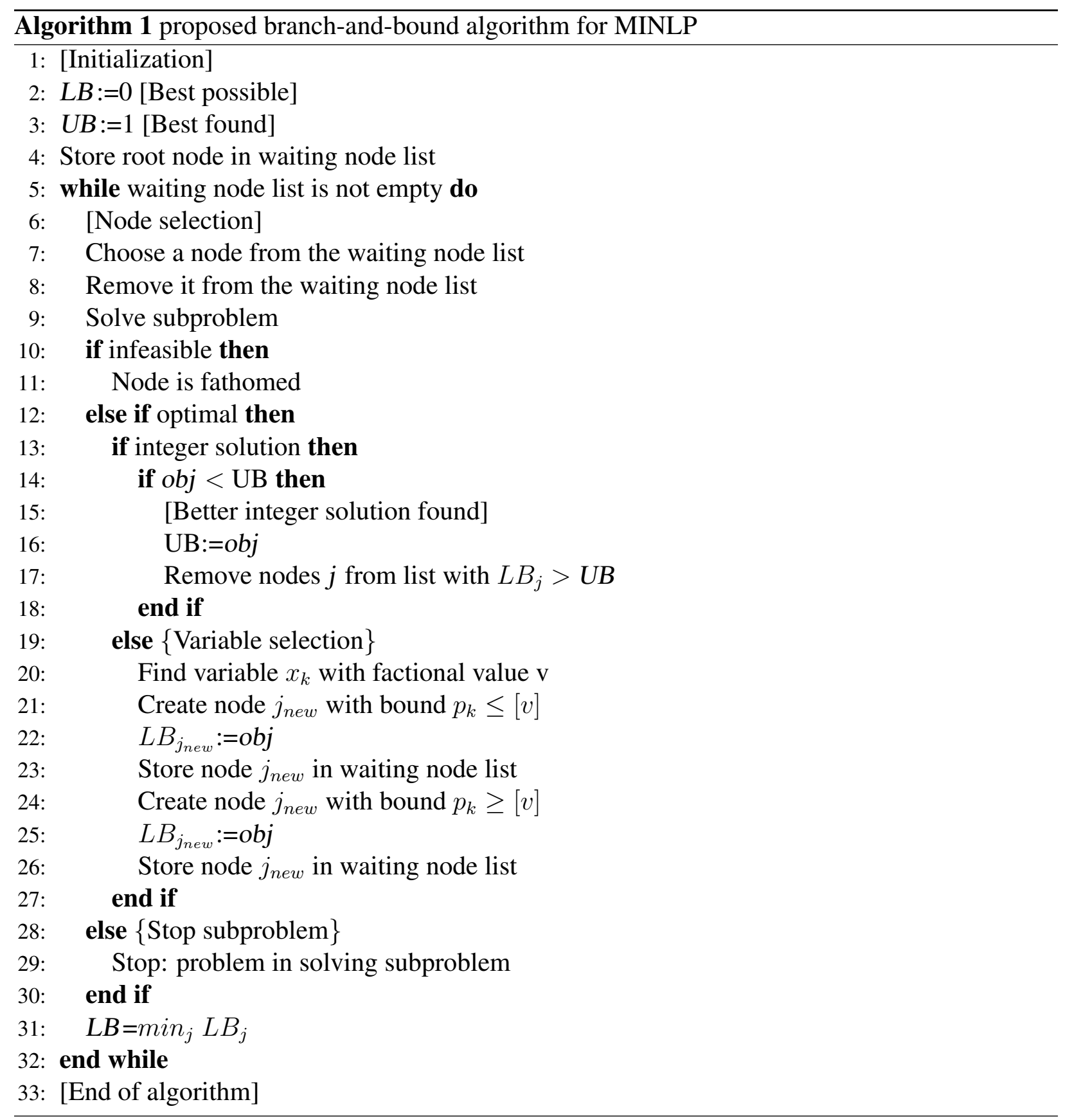


When coding the program, we propose to use the following algorithm, Algorithm 2. In this

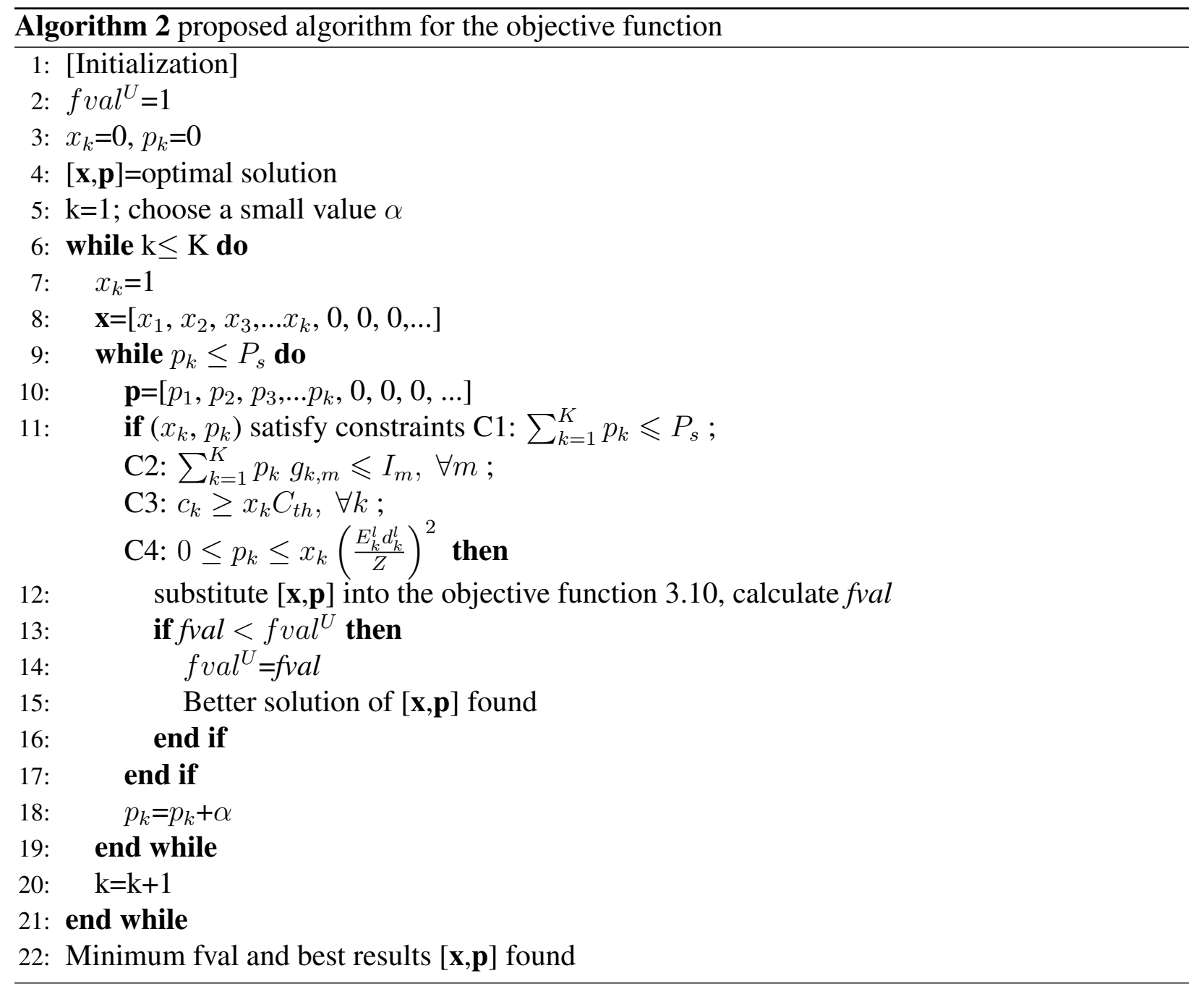

algorithm, $f_{v a l}{ }^{U}$ is the upper bound of $f v a l$, which is one. $[\mathbf{x}, \mathbf{p}]$ is a vector of feasible solution. Initial guess for $x_{k}$ and $p_{k}$ are zeros. We choose a small value of $\alpha$, which is less than $10^{-8}$. For each case, we have different $K$ 's value. By doing the while loop, we find many feasible solutions when they satisfy the constraints $\mathrm{C} 1$ to $\mathrm{C} 4$. We compare the function value fval to find the minimum one, which is the best solution. Therefore, we obtain the best result for each case. Also, we use those results to calculate other related values such as total transmitting power, throughput and number of admitted users in order to compare and analyze them in greater detail. 


\subsection{Chapter Summary}

In this chapter, we firstly introduced the structure of the single floor in hospital designed by us. Then we described our communication wireless network environment in details. Secondly, we formulated our problem by taking three factors into account, which are admission control, power allocation control and throughput guarantee. The final formulation is a multi-objective non-convex mixed integer non-linear programming (MINLP) problem. We used weighted sum method for this multiple objective optimization problem with $w_{1}, w_{2}, w_{3}$ being different weights. At last, we gave a brief introduction for MINLP, OPTI Toolbox and the proposed branch-and-bound algorithm that we used to solve our problem. 


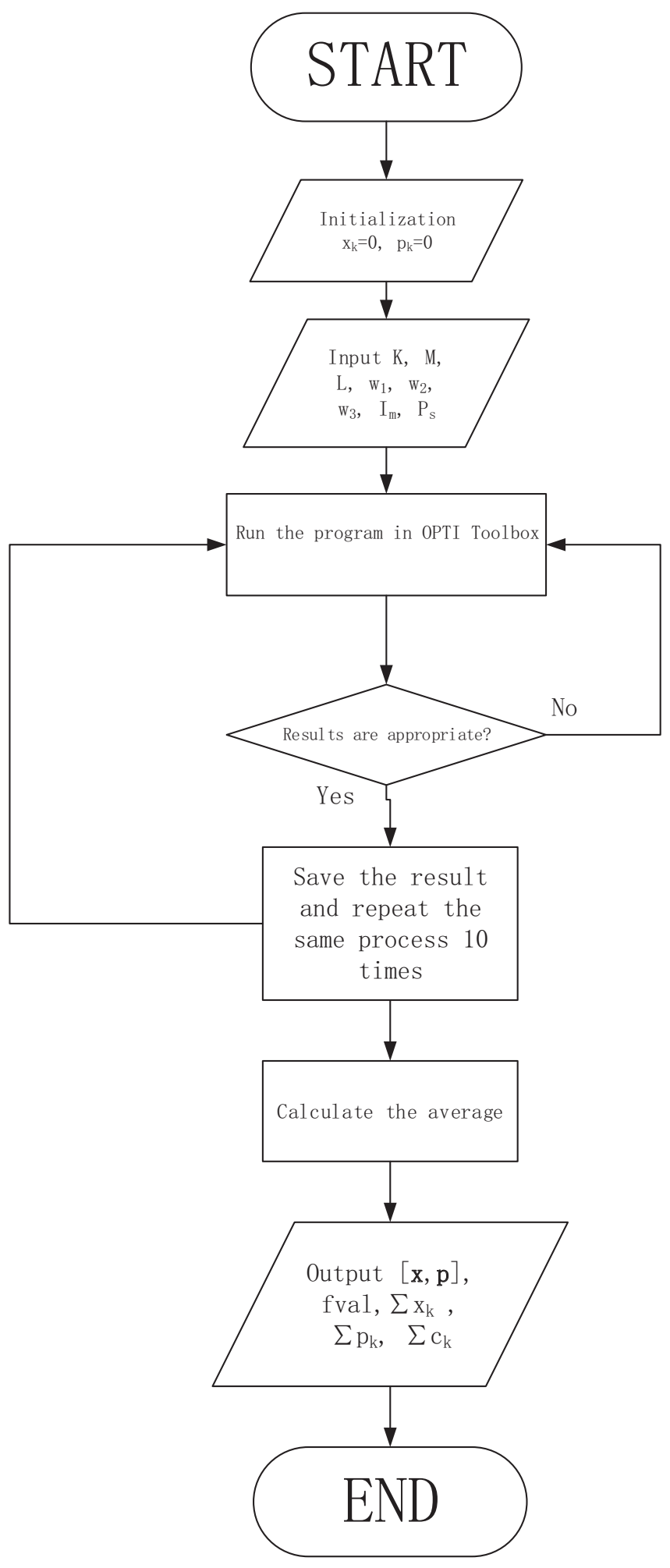

Figure 3.3: Flow Chart 


\section{Chapter 4}

\section{Simulation and Results}

In this chapter, we firstly assign appropriate values to all notations given in Table 3.1. We also explain why these values are assigned for each variable. After that, we run the program coded in MATLAB to solve the objective function (3.11) with the proposed algorithm described in the last chapter. At last, we record all the results and analyze them.

\subsection{Value Assignment}

Firstly, we assign values to all notations including constants and variables. In this thesis, we consider a medium scale hospital infrastructure. According to our Figure 4.1, the number of secondary users in one floor is equal or less than 10 . Therefore, the number of secondary user $K=2,3,4 \ldots 10$. The number of protected users and primary users is assumed to be less than half of the number of secondary users because they do not always transmit signals. Privileged users are in the minority in general. Therefore, the number of primary and protected users is $M=L=1,2,3,4,5$.

We suppose that our medical devices/users are operating with low level of power consumption. For example, a device named WIMD (wireless implantable medical device) is introduced in [51]. During their entire using period of up to 10 years, they are operated by batteries. In the WIMD design, ultra-low power consumption is a primary objective. Some of recent WIMDs are designed to generate stable frequency consuming very low power, which is even below $100 \mu \mathrm{W}$ [52]. We 
Table 4.1: Notational Values

\begin{tabular}{|l|l|l|}
\hline Symbol & Meaning & Value \\
\hline$K$ & Number of secondary users(SUs) & $2-10$ \\
\hline$M$ & Number of primary users(PUs) & $1-5$ \\
\hline$L$ & Number of protected users & $1-5$ \\
\hline$P_{s}$ & Maximum of source power & $1 \mathrm{~mW}=10^{-3} \mathrm{~W}$ \\
\hline$P$ & Default Value of $P_{s}$ & $10^{-3}$ \\
\hline$X$ & A constant in grams/watt & 655 \\
\hline$E^{\text {max }}$ & $E^{\text {max }}=X P_{s}$ & 0.655 grams \\
\hline$W$ & Channel bandwidth & $10 \mathrm{kHz}=10^{4} \mathrm{~Hz}$ \\
\hline$C^{\text {max }}$ & Maximum of $c_{k}$ & $10^{4} \log _{2}\left(10^{5}\right)$ \\
\hline$C_{t h}$ & Minimum rate requirement & $10^{4} \log _{2}\left(10^{2}\right)$ \\
\hline$h_{k}$ & The channel from $k_{t h}$ SU to CPP & Random variable \\
\hline$h_{k}^{\text {max }}$ & The best channel gain from $k_{t h}$ SU to CPP. & 1 \\
\hline$N_{0}$ & Noise power & $10^{-8} \mathrm{~W}$ \\
\hline$g_{k, m}$ & The channel from $k_{t h}$ secondary user to $m_{t h}$ PU & Random variable \\
\hline$I_{m}$ & Maximum of allowed interference at the $m_{t h}$ PU & $10^{-4} \mathrm{~W}$ \\
\hline$I$ & Default Value of $I_{m}$ & $10^{-4}$ \\
\hline$w_{1}, w_{2}, w_{3}$ & Weights, $w_{1}+w_{2}+w_{3}=1$ & variables \\
\hline$E_{k}^{l}$ & The electric field from SU $k$ to a protected device $l[44]$ & $0.1 \mathrm{~V} / \mathrm{M}$ \\
\hline$d_{k}^{l}$ & The distance between the PU $l$ and the SU $k$ [44] & 9 meters \\
\hline$Z$ & Constant from the free-space impedance of protected device $l[44]$ & $15 \mathrm{~W}$ \\
\hline
\end{tabular}

can assume that a regular small ion-lithium battery can store at most 3000 Joules of energy [51], after a quick calculation, we conclude that the maximum power consumption of one WIMD is $10 \mu \mathrm{W}$. However, we need to increase this power level a little bit since a WIMD needs to process digital sigals and wireless transmissions many times everyday in order that doctors have the latest information about the patients. Thus, we increase this power level by 10 times to be $0.1 \mathrm{~mW}$. Given the number of secondary users in our figure, $P_{s}=1 \mathrm{~mW}=10^{-3} \mathrm{~W}$.

Constant X has different values when different source of electricity is used. X equals 370 , 640, 670, 940 for natural gas, crude oil, diesel oil and lignite/brown coal, respectively. In this thesis, we calculate the mean value of those four numbers to evaluate the value of $\mathrm{X}$, which is 655 . Therefore, $E^{\max }=X P_{s}=655 \times 10^{-3}=0.655$ grams.

Given that wireless signals are highly likely to go through patients' bodies, a data rate of less than 200kbps does no harm to human being when its sampled and processed around human bodies. Hence, we set our medical device's frequency to be between $10 \mathrm{kHz}$ and $20 \mathrm{kHz}$, which means our channel bandwidth $W$ is $10 \mathrm{kHz}$.

Noise power in wireless transmission in hospital is generally caused by electrical patient trans- 
portation cars, electronics products' chargers and computer networking cabling. In [53], a noise power density for an isotropic antenna during a wireless application transmission using Bluetooth or WLAN is measured. The noise power density figure shows that most of time the noise power is around $-80 \mathrm{~dB}$, which means the noise power $N_{0}=10^{-8} \mathrm{~W}$.

$C^{\max }$ is the maximum of $c_{k}$, when user $k$ is using all the power allowed in the system which is $10^{-3} \mathrm{~W}$, with the best channel gain $h_{k}^{\max }=1$. Then, we can calculate its value, which is $10^{4} \times$ $\log _{2}\left(10^{5}\right)$. Similarly, we calculate our minimum rate $C_{t h}$ to be $10^{4} \log _{2}\left(10^{3}\right)$ based on our mimimum power and channel possible, and we lower its value further to avoid any conflict in MATLAB program. $h_{k}$ and $g_{k, m}$ are channels with random values between 0 and 1 with uniform distribution. $I_{m}$ is equal or less than $10^{-4} \mathrm{~W}$.

We use the information provided in [54] for hospital size and layout to calculate the size of our hospital floor. The hospital community requires to share some of functions as long as the size and layout is safe enough to protect patients' lives. For dining, entertainment and more spaces, one bed for patient must have at least 5.11 square meter. The number of patent rooms in the nursing unit should be less than 25. Also mentioned in [54] that in a shared-bedroom must have a minimum room area of 9.29 square meters per bed in order to be functional. Therefore, the size of our hospital is calculated based on above data. For instance, there are four beds occupying half of our protected area. We consider 10 square meters to be a reasonable size for one bed area, thus we have $10 \times 4=40$ suqare meter area for half of protected area. According to the shape of our hospital, the width should be 10 meters while the length is about 50 meters. At last, we can simply estimate the $d_{k}^{l}$ to be 9 meter.

$Z$ is a constant from the free-space impedance of protected device $l$, with the unit of $W$ [44]. Authors in [44] define $Z$ between $800 \mathrm{MHz}$ to $2.5 \mathrm{GHz}$ equal to $7 \sqrt{W}$ and $23 \sqrt{W}$ for nonlifesupporting devices and life-supporting devices, repectively [44].

$E_{k}^{l}$ is the electric field from secondary user $k$ to a protected device $l$. Authors in [55] report that from 2009 to 2011, researchers have done over one thousand measurements for electric fields in all kinds of areas within hospitals. The result of the mean value of electric field is $0.31 \mathrm{~V} / \mathrm{M}$. 
Although modern mobile medical devices bring us such a convenience and improve the efficiency of healthcare services, the electric field caused by those devices still do a lot harm to both of our patients and healthcare staff who are constantly exposed to the electric field. Thus, to reduce the electric field value to a lower level has been always a research effort. In common area, maximum of the electric field value $E$ equals $2.14 \mathrm{~V} / \mathrm{M}$ and the mean value of it is $0.19 \mathrm{~V} / \mathrm{M}$ [55]. For other areas in hospitals, such as outside and emergency department, the average value of electric field are 0.25 and $0.23 \mathrm{~V} / \mathrm{M}$, respectively [55]. In this thesis, the floor of our hospital is a common area and we want to reduce the radiation of the electric field, therefore we set the value of $E$ to be $0.1 \mathrm{~V} / \mathrm{M}$.

In the next section, we substitute these assigned values to the variables in our function and run the coded program in MATLAB to find the minimum function value. Also, we record all related results such as total power, throughput and number of admitted users and plot them in next section.

\subsection{Results}

We start with the simplest case with two secondary users in the hospital to simulate our problem. As illustrated in Figure 4.1, we first consider a small group of users in the hospital which includes one protected user, one primary user and two secondary users. Thus, in this case, $K=2, M=1, L=1$. As Figure 4.1 shows, the CPP is located in the center of this floor on the ceiling. Of two secondary users, one is in the large nursing unit, the other is in the protected area along with the only one protected user. Primary user is also in the large nursing unit but he is far away from the secondary user in this unit. When the number of different kind of users increase for different cases, all three types of users' locations do not change, as located in Figure 4.1.

Meanwhile, we start with equal weights in (3.11) for three different parts of our objective function. Then, we set one of the three weights to be one and others to be zero, which means we do only one control method each time (i.e., call admission control, green power allocation and throughput guarantee). Later, we simulate our program with single dominant weights. In equal weight and unequal weight case, we also change the values of $I_{m}$ and $P_{s}$ and study the difference 


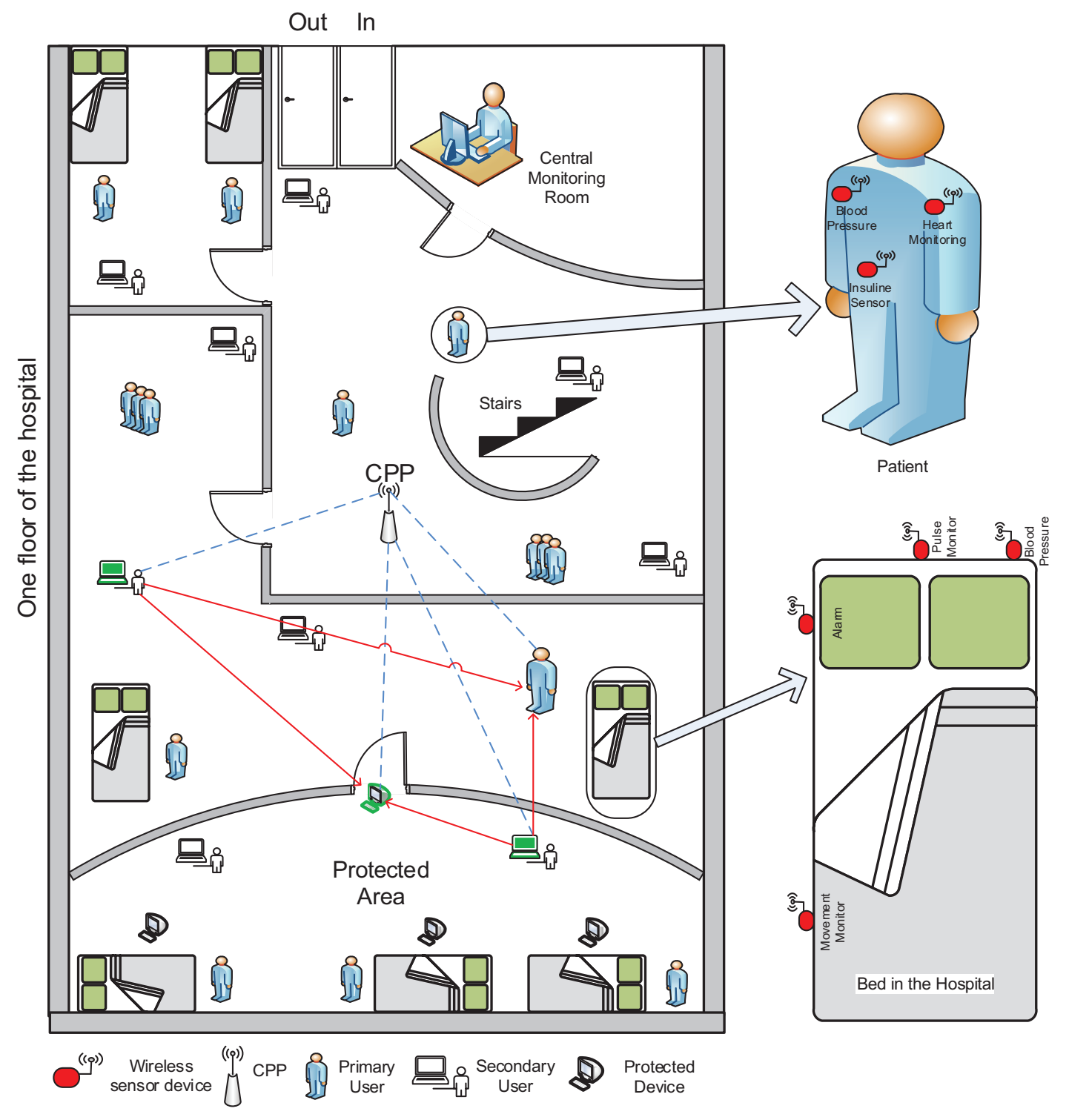

Figure 4.1: A single-floor system model 
in performance.

After running our program, we get the values of vector $\mathbf{x}$ and $\mathbf{p}$ such that we know how many users are admitted and not-admitted, and how much power each user is transmitting. Also the function value and throughput will be shown. We record all those results and plot them. Then, we increase the number of secondary users $K$ and number of primary users $M$ and number of protected user $L$, before running the program again. By doing so, we get required results in order to analyze them. We use the following user configuration:

Table 4.2: User Configuration

\begin{tabular}{|l|l|l|l|l|l|l|l|l|l|}
\hline$K=$ & 2 & 3 & 4 & 5 & 6 & 7 & 8 & 9 & 10 \\
\hline$M=$ & 1 & 1 & 2 & 2 & 3 & 3 & 4 & 4 & 5 \\
\hline$L=$ & 1 & 1 & 2 & 2 & 3 & 3 & 4 & 4 & 5 \\
\hline
\end{tabular}

\subsubsection{Equal Weight Case}

Firstly, we start with equal weights scenario where $w_{1}=w_{2}=w_{3}=\frac{1}{3}$. We have four figures showing number of admitted users, total power, function value and secondary user throughput respectively with different values of interference, $I_{m}$. Similarly, we have another set of four figures showing the same with different values of total power allowed in the system, $P_{s}$. And we show the same figures for other cases in next sections.

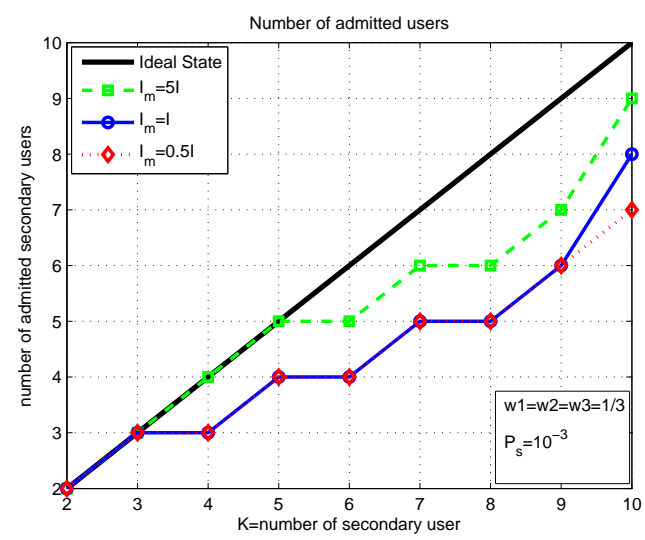

Figure 4.2: Number of admitted users when $I_{m}$ changes

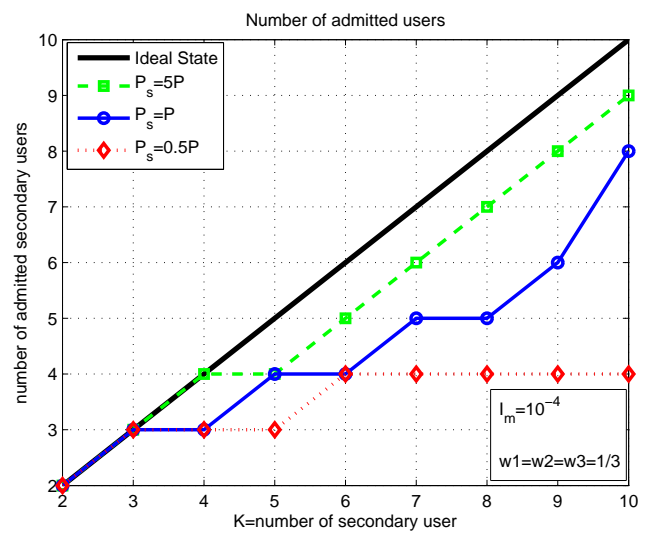

Figure 4.3: Number of admitted users when $P_{s}$ changes 
Figure 4.2 shows the number of admitted users with different $I_{m}$. Here, all weights are $1 / 3$ and $P_{s}$ equals $10^{-3}$. And all results are averaged over 10 iterations of running the program. The bold line represents ideal state in terms of maximum admitted users, the normal line represents our results when $I_{m}$ equals $I$, the dash line represents results when $I_{m}$ equals $5 I$, the dotted line represents results when $I_{m}$ equals $0.5 I$, where $I$ is the default value of $I_{m}$ which is $10^{-4}$.

In ideal state, all secondary users can be admitted to transmit. When $I_{m}$ equals its default value of $I$, the number of admitted user is $2,3,3,4,4,5,5,6,8$ respectively for $K=2,3,4, \ldots 10$. As we can see, all users can be admitted when we have two to three users waiting to communicate, but the number of admitted users does not increase linearly when we have four or more users. More secondary user transmission increases the interference to primary users or protected users. The number of admitted users stay the same when number of primary and protected users increase by one because our constraint $\mathrm{C} 2$ limits the interference level. This can be explained by our formulation, our function's constraints C2 and C3 limit the interference level and also ensure a minimum threshold of throughput of secondary users. When number of primary and protected users increases, the interference increases, at the same time it goes over its maximum value $I_{m}$, so each user's power $p_{k}$ has to be lowered to satisfy constraint C2. On the other hand, constraint C3 makes the single user's required power to be greater to meet the capacity threshold, which means the power $p_{k}$ has to be increased but it is limited. Therefore, the best solution is to limit the number of users.

When $I_{m}$ equals 5I, we increase the value of interference threshold, thus we can have more number of users admitted comparing to the normal case. The number of admitted users is a little higher than the number when $I_{m}$ equals default value $I$. The dotted line is very similar to the normal line which indicates that number of users doesn't change too much when we lower the interference level $I_{m}$. Similar situation is noted when we change the value of maximum source power $P_{s}$ with fixed $I_{m}=10^{-4}$ as Figure 4.3 shows. When $P_{s}$ increases to $5 P$, it impacts the constraint $\mathrm{C} 1$ in (3.12) resulting in more number of users admitted and more power $p_{k}$ allowed.

Figure 4.4 shows total transmit power of secondary users. When $I_{m}$ equals $I$, the total power 


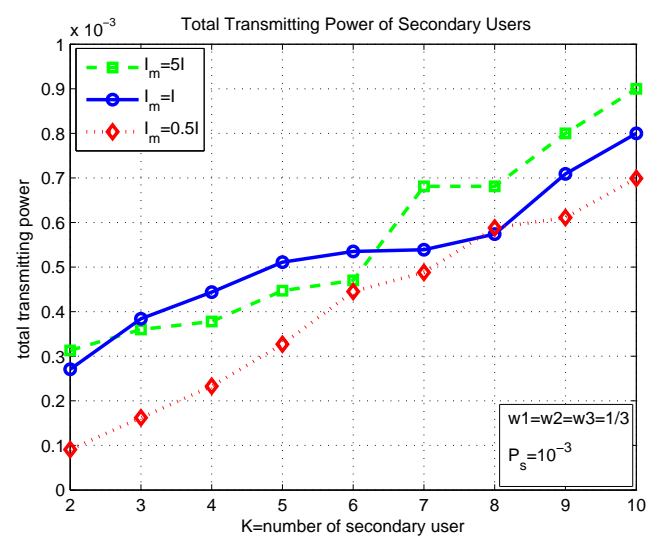

Figure 4.4: Total transmitting power (Watt) of all secondary users when $I_{m}$ changes

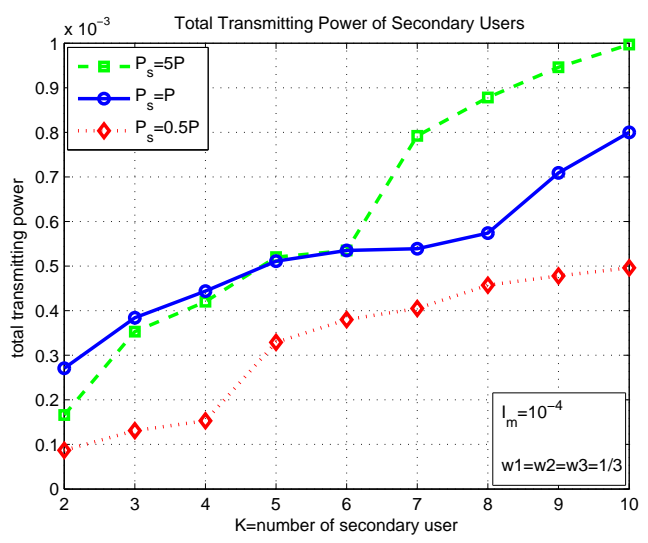

Figure 4.5: Total transmitting power (Watt) of all secondary users when $P_{s}$ changes

increases steadily from $2.71 \mathrm{E}-04$ to $8.00 \mathrm{E}-04$ as the number of admitted users increases. This means that more power has to be allocated to allow more secondary users to transmit together. When $I_{m}$ equals $5 I$, total power remains at the same level when there are less than 6 users. When it is above 6 , the total power increases rapidly to a higher level than the normal level, which means more transmitting power is allowed since we increase the interference level $I_{m}$. When $I_{m}$ equals $0.5 I$, the curve has similar trend as the normal case but the value of it is much lower since we lower the interference level $I_{m}$ which impacts the constraint $\mathrm{C} 2$.

Similar situation is noted in Figure 4.5 with different values of $P_{s}$. When $P_{s}$ equals $5 P$, the total power can go up to $10^{-3}$ Watt which is much higher than previous results since we increase the maximum source power $P_{s}$. Next, Figures 4.6 and 4.7 show the function value, $f v a l$, which was

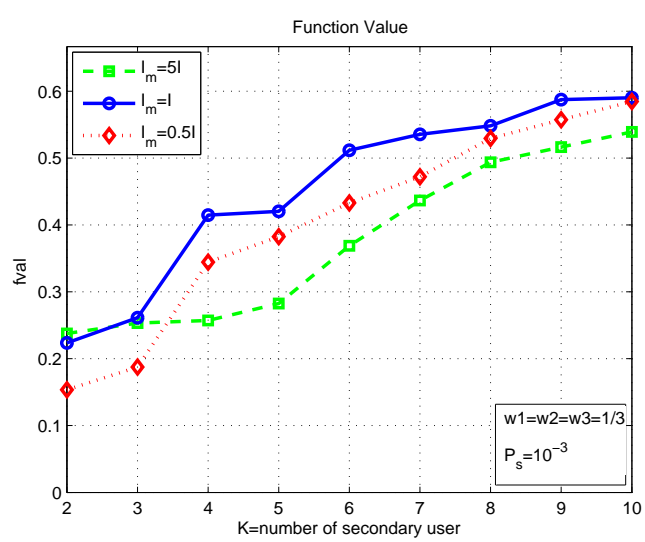

Figure 4.6: Function value when $I_{m}$ changes Figure 4.7: Function value when $P_{s}$ changes

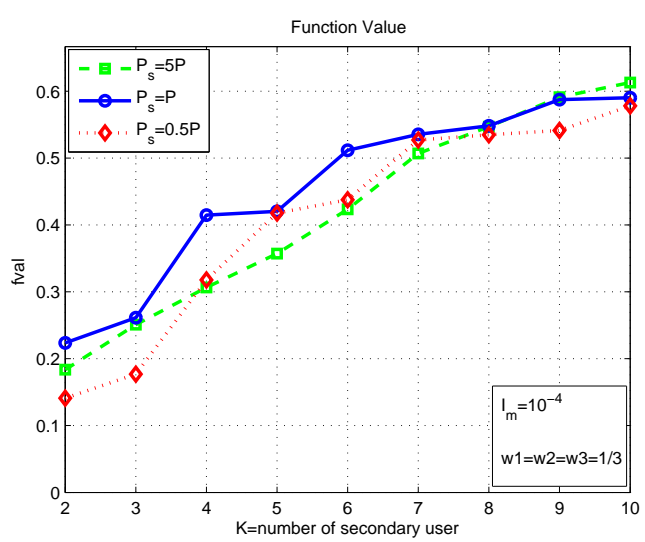


defined in (3.16). As all three lines indicate, the function value fval increases steadily form 0.15 to 0.6 as the number of users increase. The maximum of function value is expected to be 0.667 , because it is the value when all $\mathbf{x}$ equals zero which means no users are active. To minimize the function value is our final goal in our work, so the function value is a good indicatior of our effort in solving the MINLP problem. It means that as the number of admitted users increase, it is more and more difficult to reach the minimum value of the whole function. Thus, it is harder to achieve our minimizing objective.

Both the dash and dotted lines are lower than the normal line which is an interesting result. The reason is that more interference level allows more number of users which decrease the first part of our function, and less interference level limits the total power which decreases the second part of our function.

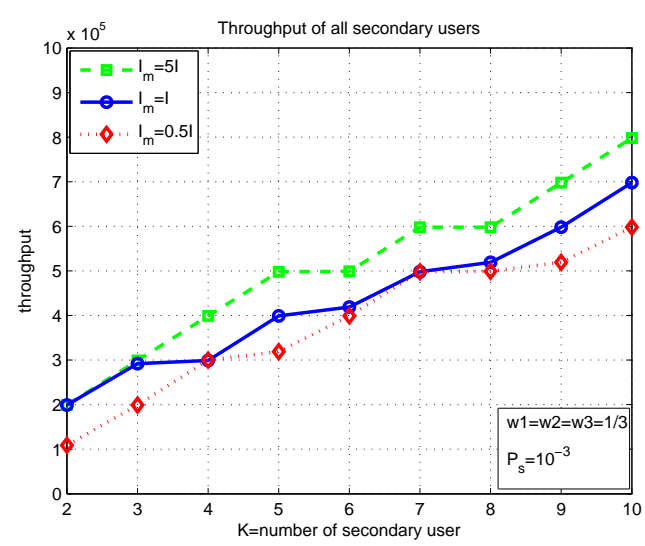

Figure 4.8: Throughput (bps) of all secondary users when $I_{m}$ changes

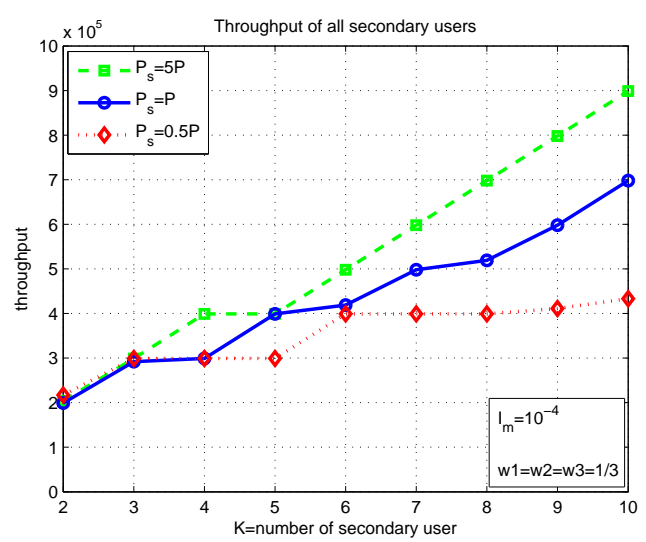

Figure 4.9: Throughput (bps) of all secondary users when $P_{s}$ changes

At last, Figure 4.8 and 4.9 show throughput of all secondary users. Throughput goes from $1.99 \mathrm{E}+05$ to $6.98 \mathrm{E}+05$ steadily as the normal line shows. And it is bit higher and lower when $I_{m}$ and $P_{s}$ change as the dash and dotted lines indicate. The throughput values do not change too much because the change of power $p_{k}$ doesn't have much impact on the value of the throughput when we put the changed $p_{k}$ into the formula. 


\subsubsection{Unequal and Dominant Weight Case}

In this section, we present our results with unequal weights assigned to admitted users, transmit power and throughput.

Only Admission Control $\left(w_{1}=1\right)$

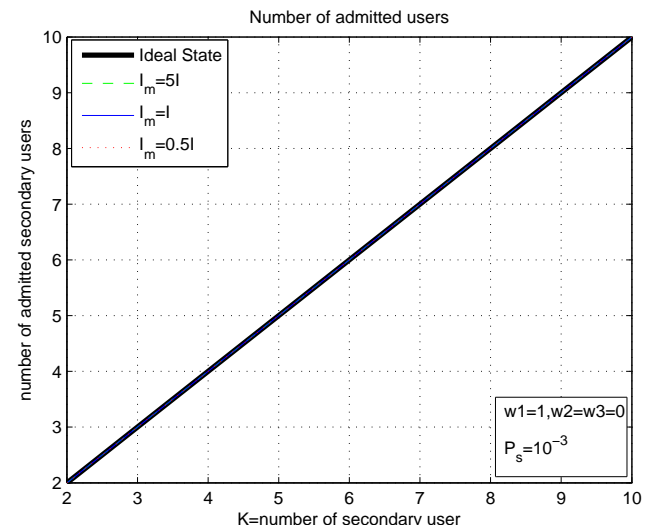

Figure 4.10: Number of admitted user when $I_{m}$ changes

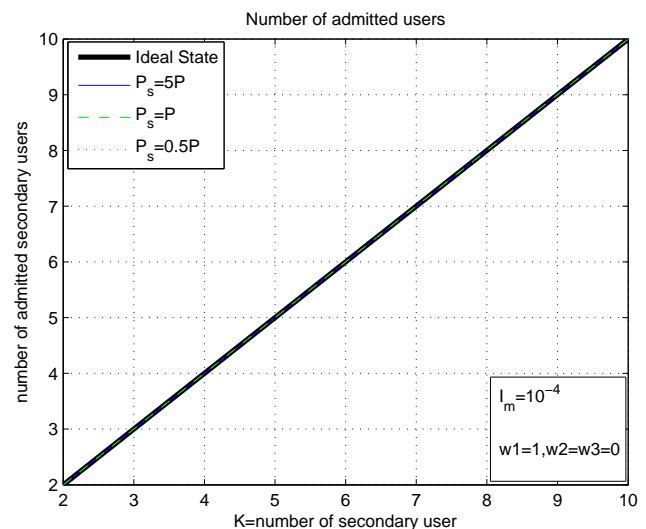

Figure 4.11: Number of admitted user when $P_{s}$ changes

In this first subsection, we set $w_{1}=1, w_{2}=w_{3}=0$, which means only admission control is considered in this case. As $w_{1}=1, w_{2}=w_{3}=0$, only the first part of our function (3.11) remains valid. Theoretically, number of admitted users $k$ is always the same as the total number of users $K$ such that the function value goes down to zero to reach the minimum. Our results show that the theory and results are in conformance as Figure 4.10 and 4.11 indicate. All three types of lines overlap with the ideal state bold line. All users can be admitted no matter how the $I_{m}$ and $P_{s}$ change. We do not calculate the result when $k$ is greater than 10 , but the reality is that the number of admitted users will reach a certain number before it stops increasing due to the constraint which limits the total power in our formulation. This situation remains the same for the next few cases.

Figures 4.12 and 4.13 show the total power in this case, where we can see that the curve increases very steeply from $0.2 \times 10^{-3}$ to the maximum source power $10^{-3}$. And that the total power reaches $0.9 \times 10^{-3}$ from the beginning when $I_{m}$ equals $5 I$. The total power even goes up to $5 \times 10^{-3}$ when $P_{s}$ changes to $5 P$. This situation indicates to us that the power and throughput can 


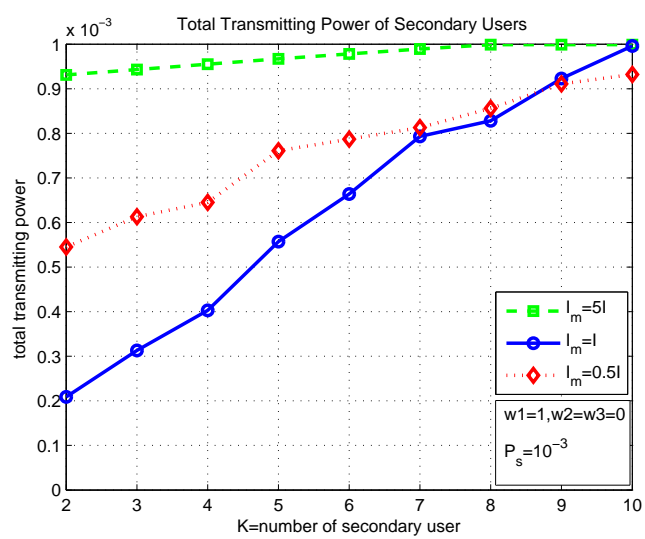

Figure 4.12: Total transmitting power (Watt) of all secondary users when $I_{m}$ changes

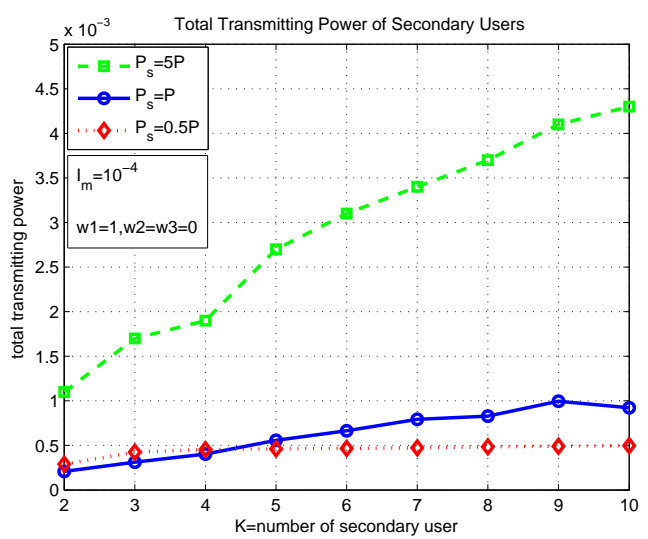

Figure 4.13: Total transmitting power (Watt) of all secondary users when $P_{s}$ changes

reach very high level in this case, since the second and the third part of our function are no longer valid in this call admission control only case. Those values can be very high as they do not impact on the function value any more. The function value is always zero because all users are admitted which makes the value of our function to be zero. Figures 4.16 and 4.17 show that throughput

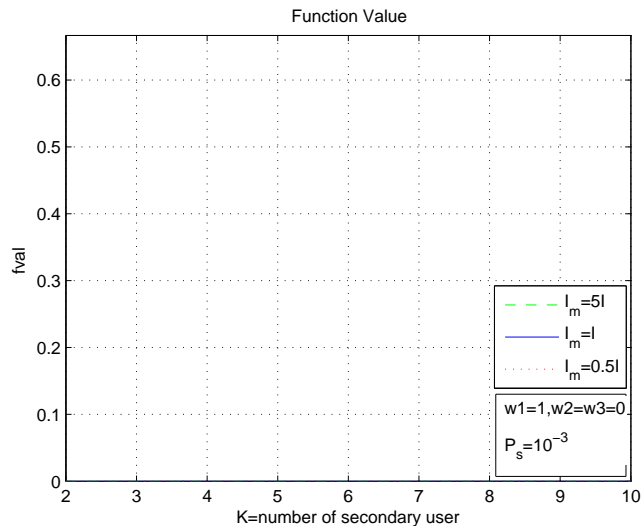

Figure 4.14: Function value when $I_{m}$ changes

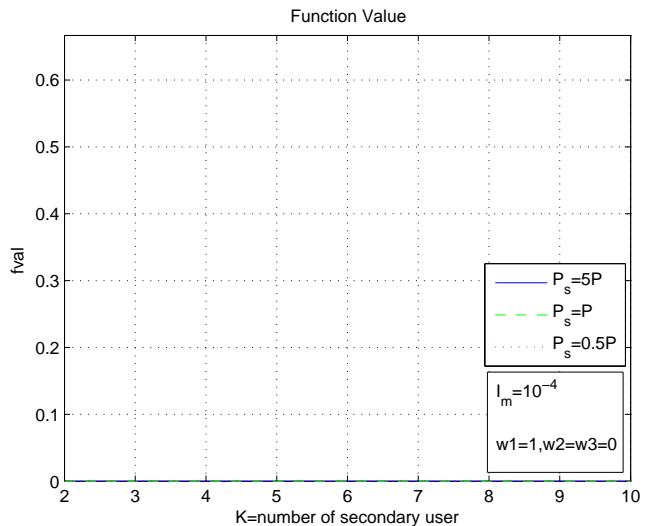

Figure 4.15: Function value when $P_{s}$ changes

in this case is slightly higher than previous one with equal weights. As we discussed before, the throughput values do not change too much because the change of power $p_{k}$ does not have much impact on the value of the throughput formula. 


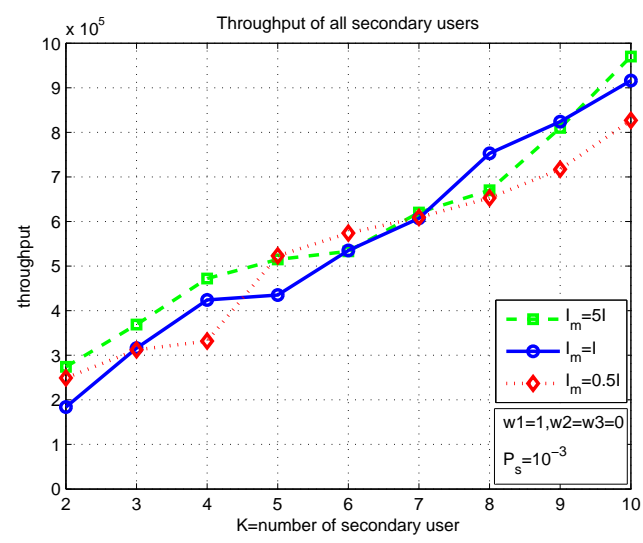

Figure 4.16: Throughput (bps) of all secondary users when $I_{m}$ changes

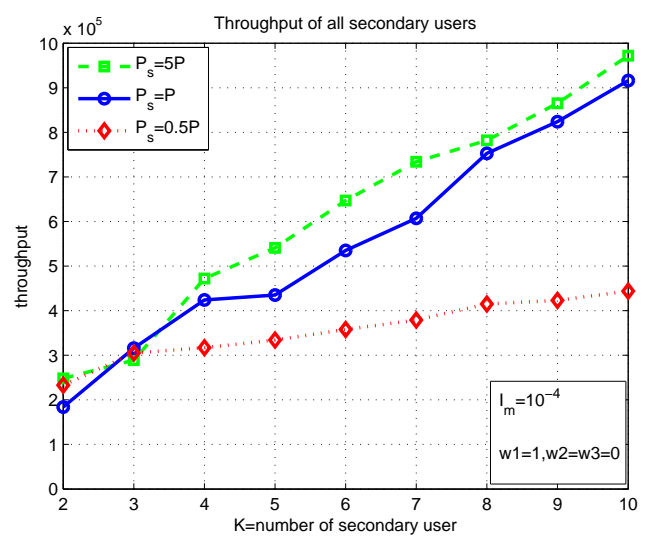

Figure 4.17: Throughput (bps) of all secondary users when $P_{s}$ changes

\section{Only Power Control $\left(w_{2}=\mathbf{1}\right)$}

In this subsection, we do power control only, which means only the second part of our function is valid. Theoretically, only power is considered, other parts of our function such as number of users and throughput are both ignored automatically. Therefore, the power of each user is minimized to reach the minimum function value objective. Figures 4.18 and 4.19 indicate that all users can be admitted as same as the only admission control section. Because the first part of our function is no longer valid, we can admit as many users as we want, which doesn't impact the function value. Figures 4.20-4.25 indicate that the total power and throughput are extremely low comparing to normal one with equal weights. The total power can be lowered to some value less than $5 \times 10^{-5}$ which is much much lower than the maximum source power. However, when number of user goes over 5 and $I_{m}$ equals $5 I$, the total power increases steeply towards $4 \times 10^{-5}$, which is much higher than those values when $I_{m}$ equals $I$ or $0.5 I$. Same situation applies when $P_{s}$ equals $5 P$ while number of users goes over 7. Because the interference level and maximum source power is 5 times larger, we can allocate more power but keep the function value low at the same time. As we can see in Figure 4.22 and 4.23, the function value is also very low since minimum power makes our function value extremely low. All function values are under $10^{-3}$ which is much lower those that in previous sections. 


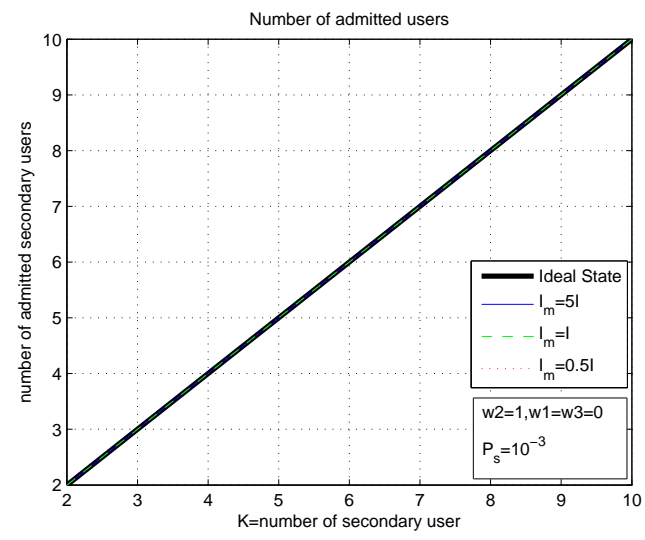

Figure 4.18: Number of admitted user when $I_{m}$ changes

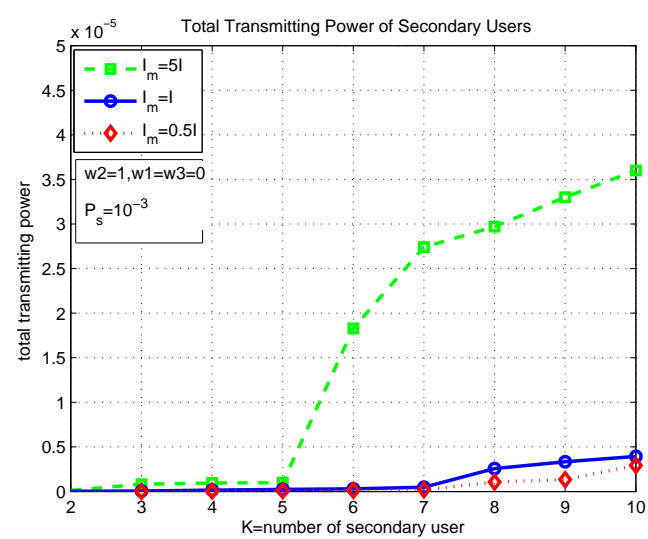

Figure 4.20: Total transmitting power (Watt) of all secondary users when $I_{m}$ changes

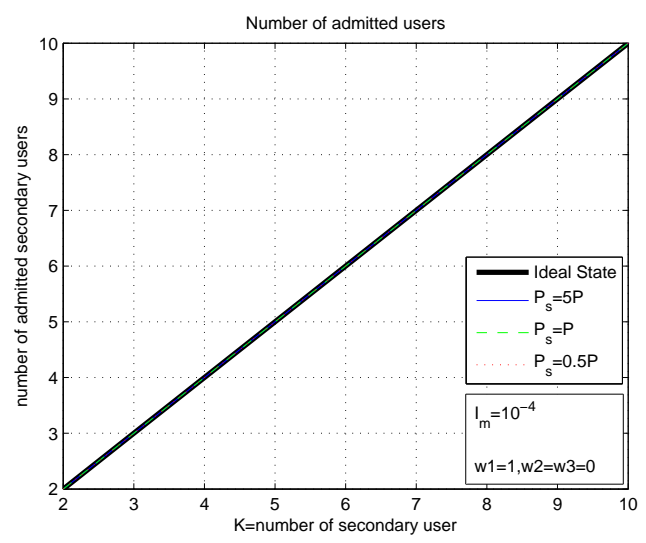

Figure 4.19: Number of admitted user when $P_{s}$ changes

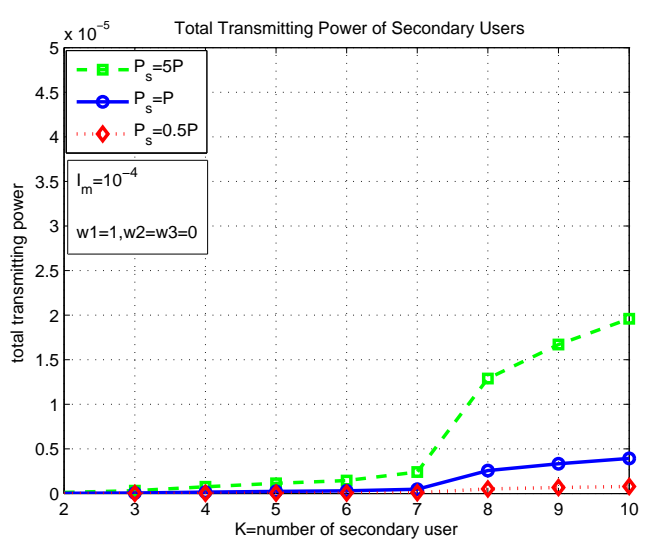

Figure 4.21: Total transmitting power (Watt) of all secondary users when $P_{s}$ changes

\section{Only Throughput Guarantee $\left(w_{3}=1\right)$}

In this subsection, we only guarantee throughput, which means only the third part of our function is valid. Theoretically, the power of each user is maximized to reach the minimum function value objective. Accordingly, the number of users and the power of each user can be as much as possible since they don't impact the function value. The situation here is totally opposite to the only power control section. Figures 4.26 and 4.27 indicate that we can still have all users admitted in the system. Figures 4.28-4.33 indicate that the total power and throughput are extremely high compared to normal one with equal weights. The total power can go up to maximum source power when number of users is greater than 5, and $I_{m}$ equals 5I. As Figure 4.29 shows, when maximum source 


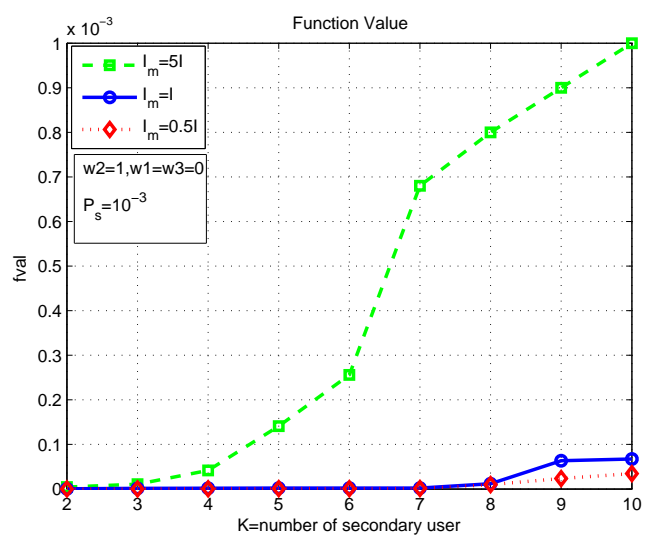

Figure 4.22: Function value when $I_{m}$ changes

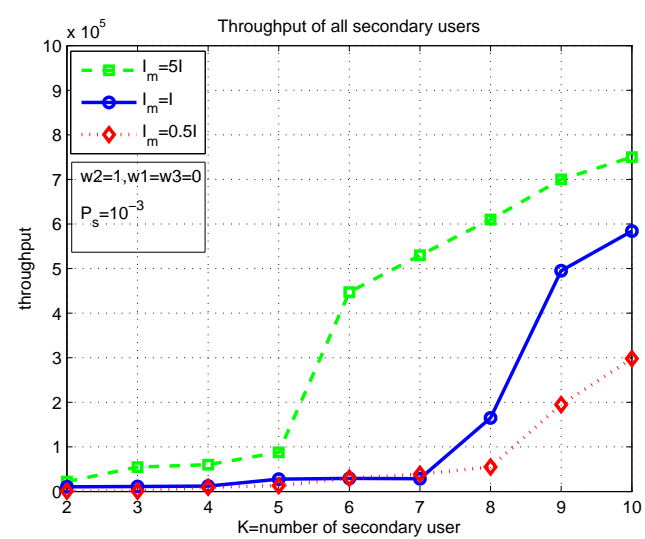

Figure 4.24: Throughput (bps) of all secondary users when $I_{m}$ changes

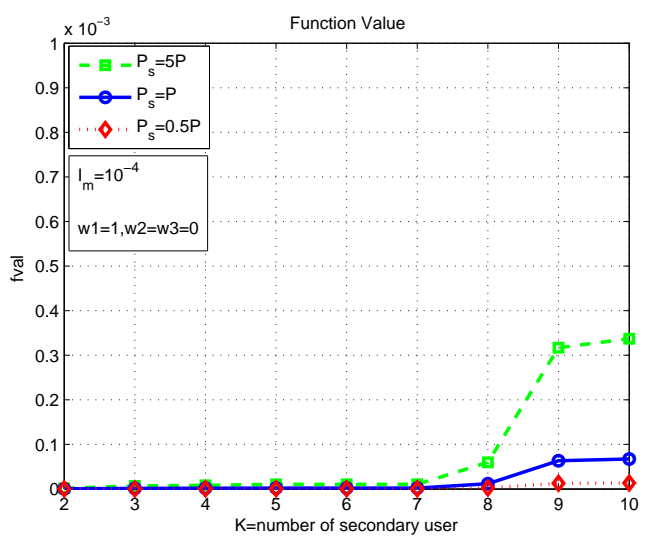

Figure 4.23: Function value when $P_{s}$ changes

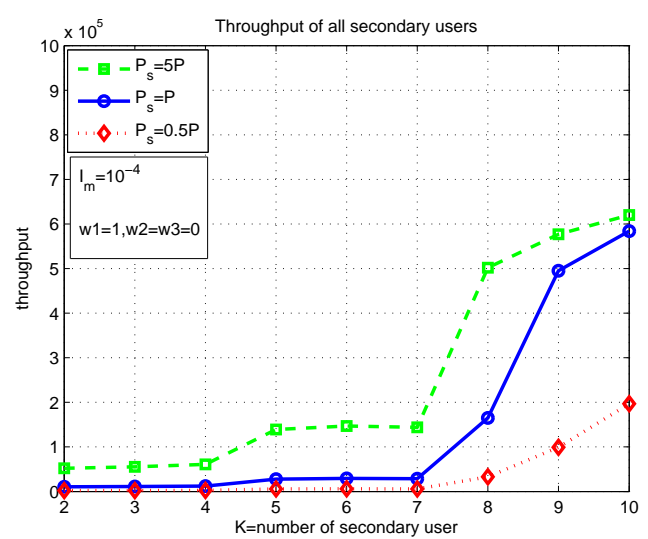

Figure 4.25: Throughput (bps) of all secondary users when $P_{s}$ changes

power $P_{s}$ changes, in each case, the total power can reach its maximum value from the beginning. All power allowed is allocated to all secondary users. The function value is also very high since more power makes our function value high. When $I_{m}$ equals $5 I$, the function value can even go over 0.6. When $P_{s}$ equals $5 P$, the function value can even go over 0.7 , which is the highest function value we ever see in all results.

\subsubsection{Unequal and Non-dominant Weight Case}

The last section is about unequal and non-dominant weight. We set one of the weights to be 0.6 , other two weights to be 0.2 , for these different cases. In the first case, $w_{1}=0.6, w_{2}=w_{3}=0.2$. With 


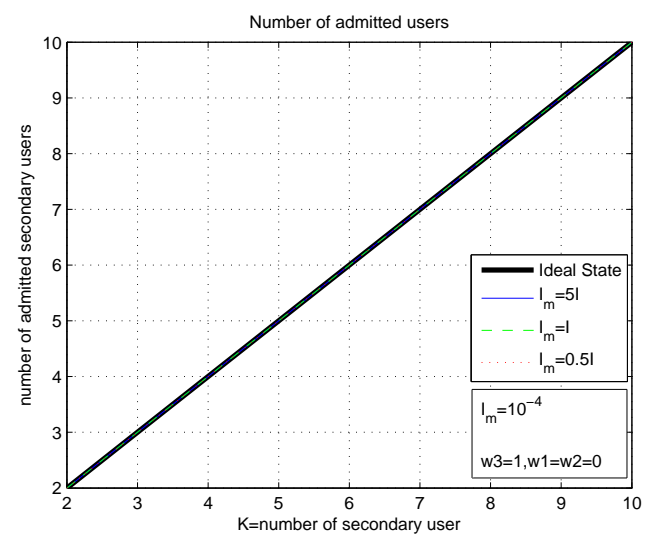

Figure 4.26: Number of admitted user when $I_{m}$ changes

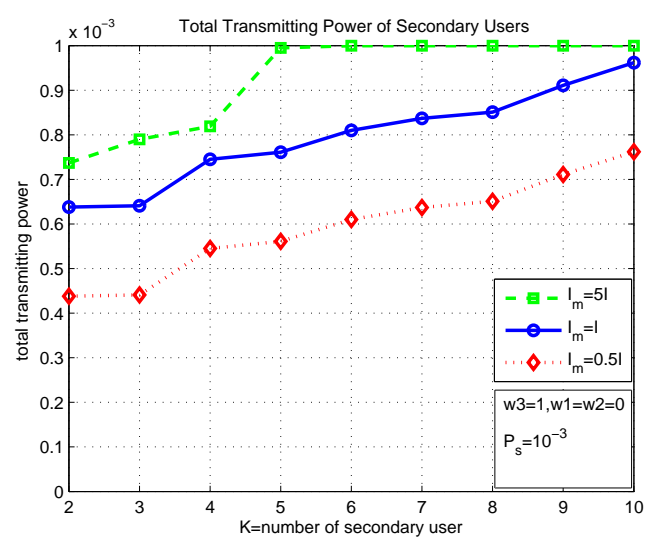

Figure 4.28: Total transmitting power (Watt) of all secondary users when $I_{m}$ changes

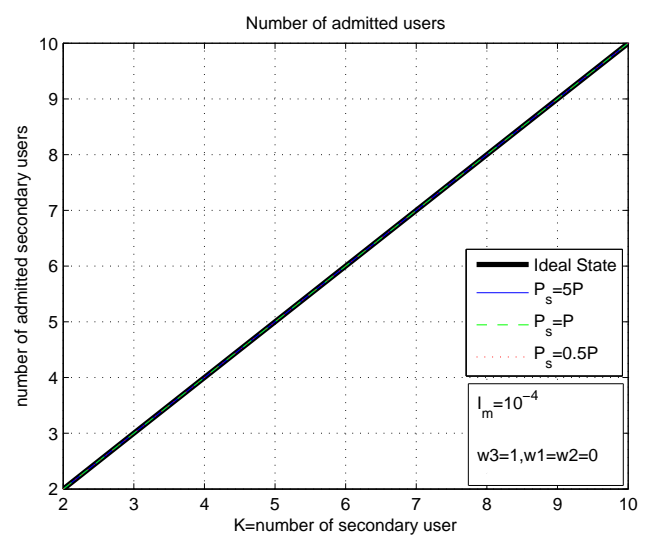

Figure 4.27: Number of admitted user when $P_{s}$ changes

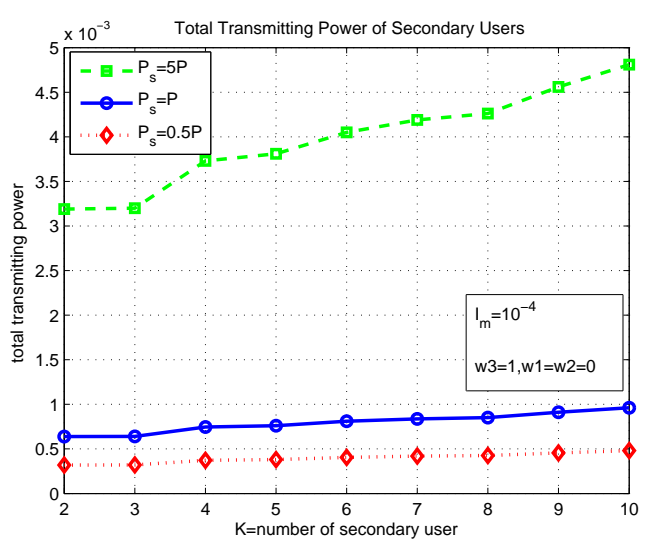

Figure 4.29: Total transmitting power (Watt) of all secondary users when $P_{s}$ changes

$w_{1}$ equals 0.6 , the number of users, total power, function value and throughput are all between those values in only admission control (with $w_{1}=1$ ) and equal weights (with $w_{1}=1 / 3$ ) as Figure 4.34-4.37 show.

The second and third cases have similar situation. When $w_{2}=0.6$, the number of users, total power, function value and throughput are all between those values in only power control and equal weights as Figures 4.38-4.41 show. When $w_{3}=0.6$, the number of users, total power, function value and throughput are all between those values in only throughput guarantee and equal weights as Figures 4.42-4.45 show. 


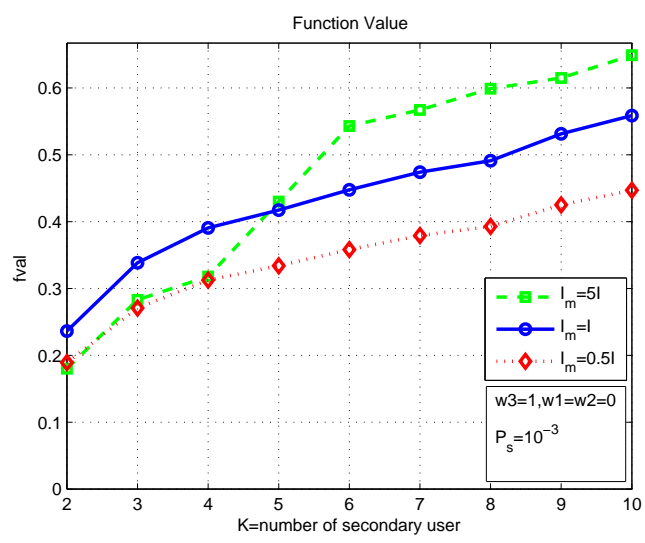

Figure 4.30: Function value when $I_{m}$ changes

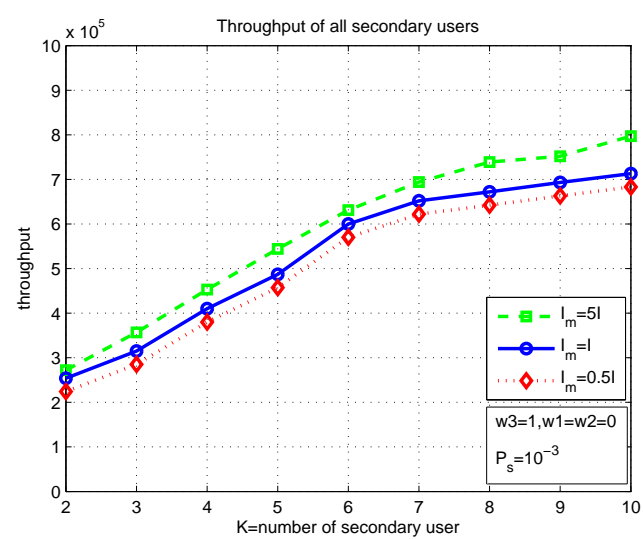

Figure 4.32: Throughput (bps) of all secondary users when $I_{m}$ changes

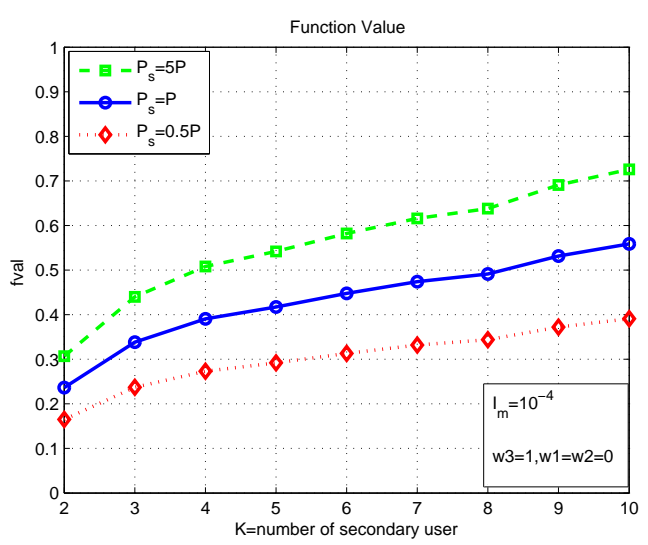

Figure 4.31: Function value when $P_{s}$ changes

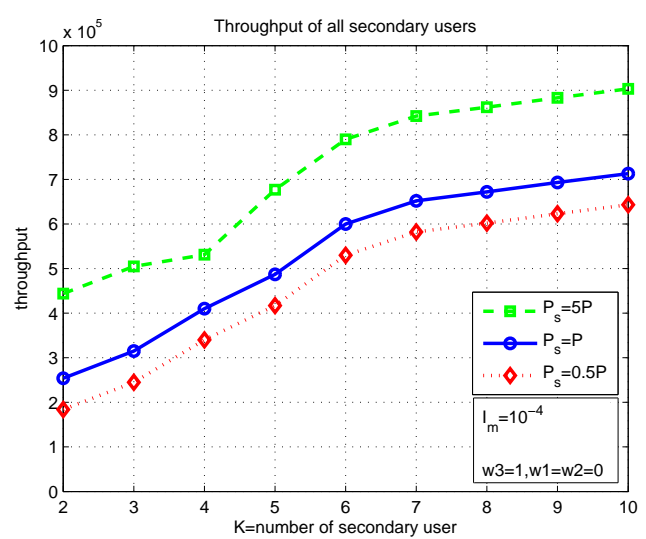

Figure 4.33: Throughput (bps) of all secondary users when $P_{s}$ changes

\section{Case 1: Dominant Call Admission Control $\left(w_{1}=\mathbf{0 . 6} w_{2}=w_{3}=\mathbf{0 . 2}\right)$}

As Figure 4.34 indicates, the bold line represents ideal state, the normal line is our experimental result in the case, where $w_{1}=0.6 w_{2}=w_{3}=0.2$. When $K$ equals 4 and 6 , the numbers of admitted users are greater by one than those in equal weight case compared to Figure 4.2. However, those numbers are much less than those in only admission control case which is also the same as ideal state compared to Figure 4.10. It indicates that when $w_{1}$ equals a value between zero and one, also greater than the values of $w_{2}$ and $w_{3}$, more secondary users can be admitted since the first part of the objective function occupies and dominates a larger proportion in the function value. Therefore, increasing number of users reduces the objective function value. 


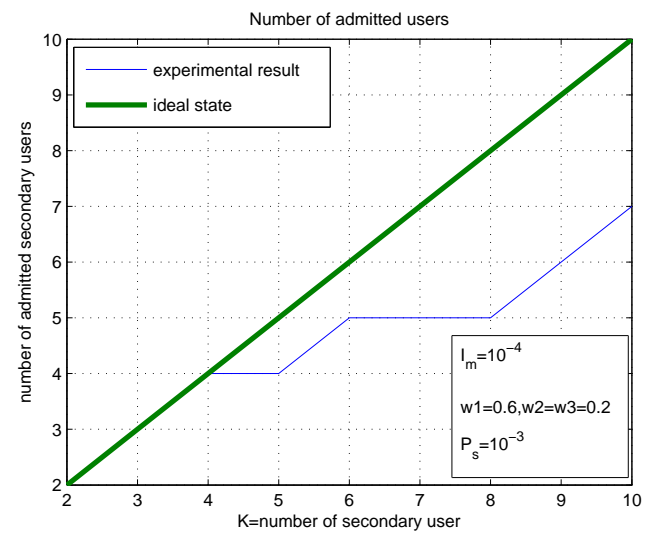

Figure 4.34: Number of admitted users

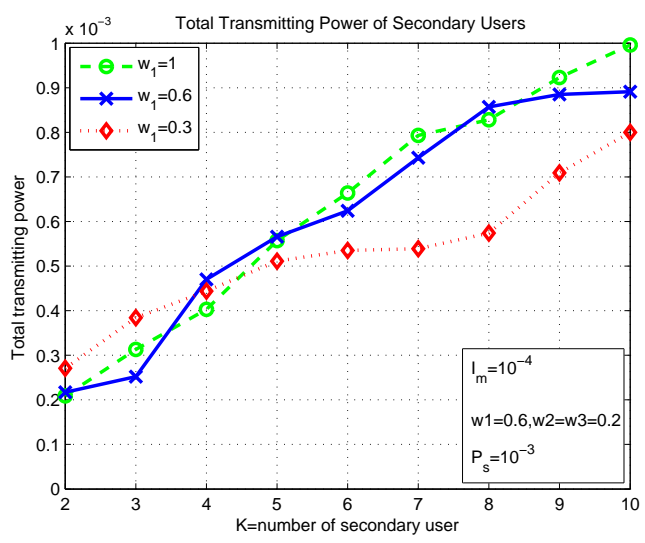

Figure 4.35: Total transmitting power (Watt) of all secondary users

Figure 4.35 shows the total power in this case. When $K$ is less than three, the total power in this case is slightly lower than that in Figure 4.4. However, when $K$ is above 4, the total power is $20 \%$ higher than that in Figure 4.4 but $8 \%$ lower than that in Figure 4.12. This indicates that more power is allocated to more users with a larger value of $w_{1}$ and smaller values of $w_{2}$ and $w_{3}$ and it is still limited because the green power control is still operating in the objective function.

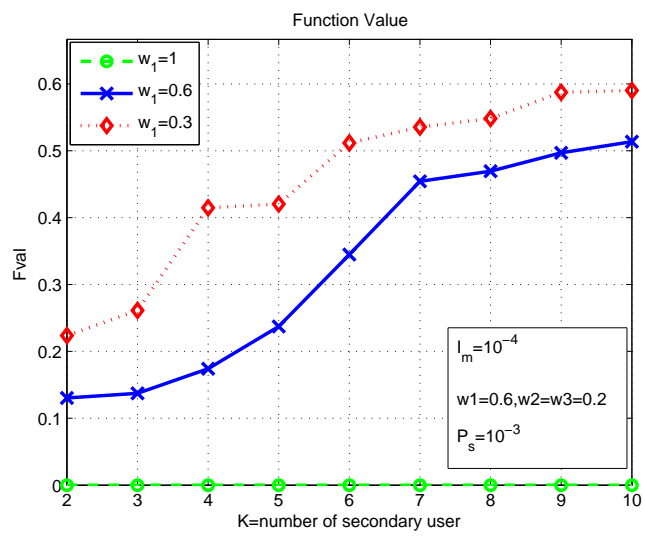

Figure 4.36: Function value

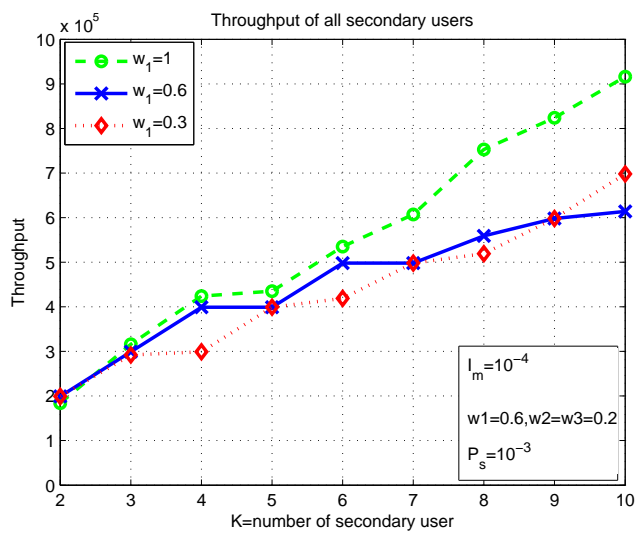

Figure 4.37: Throughput (bps) of all secondary users

The function value fval shown in Figure 4.36 is approximately the average of the $f v a l$ s in Figure 4.6 and Figure 4.14. Because the constraints and the second part of the objective function restrict the total power level, not all users are admitted as the only admission control case.

As shown in Figure 4.37, throughput in this case is similar to other cases when $K$ is under 7 . 
When $K$ is above 7, it is slightly lower than that in Figures 4.8. Because $w_{3}$ equals 0.2 which is slightly less than 0.33 in equal weight case. It causes the value of the third part of the objective function decrease since throughput guarantee is less important in this case. It is much lower than that in only admission control case when $K$ is above 6 because power is maximize in that case which leads to maximum throughput.

Case 2: Green Power Allocation $\left(w_{2}=\mathbf{0 . 6} w_{1}=w_{3}=\mathbf{0 . 2}\right)$

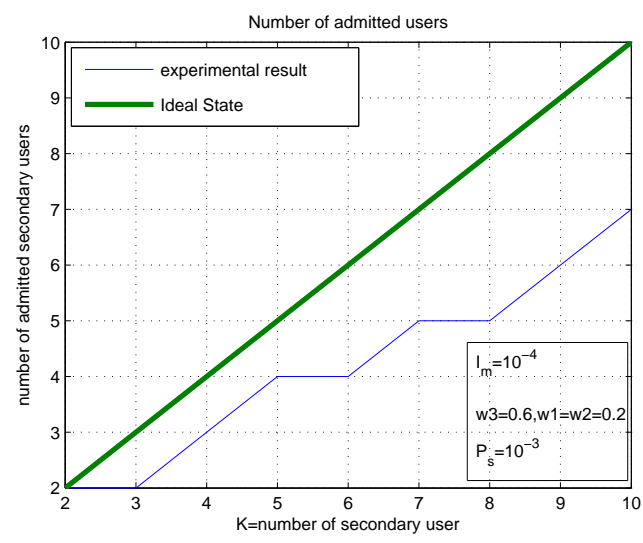

Figure 4.38: Number of admitted users

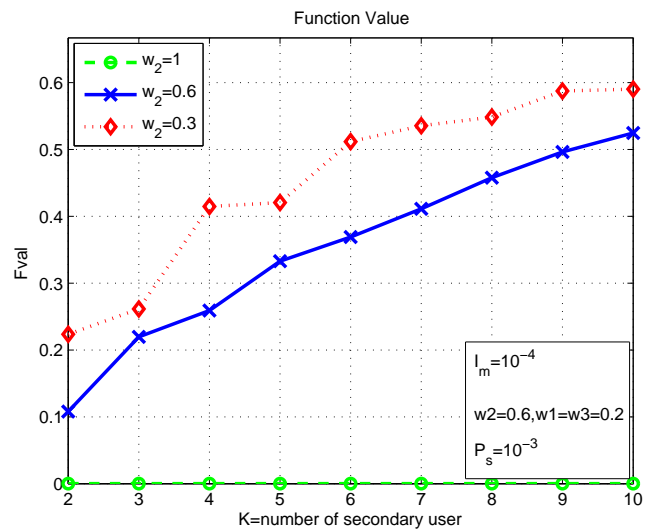

Figure 4.40: Function value

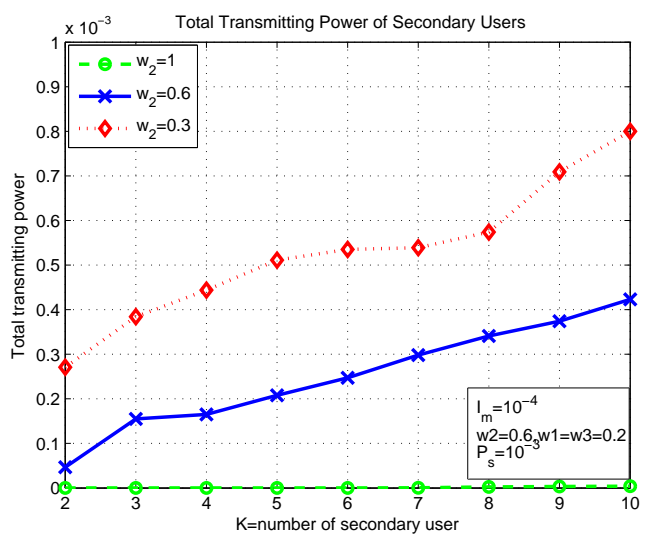

Figure 4.39: Total transmitting power (Watt) of all secondary users

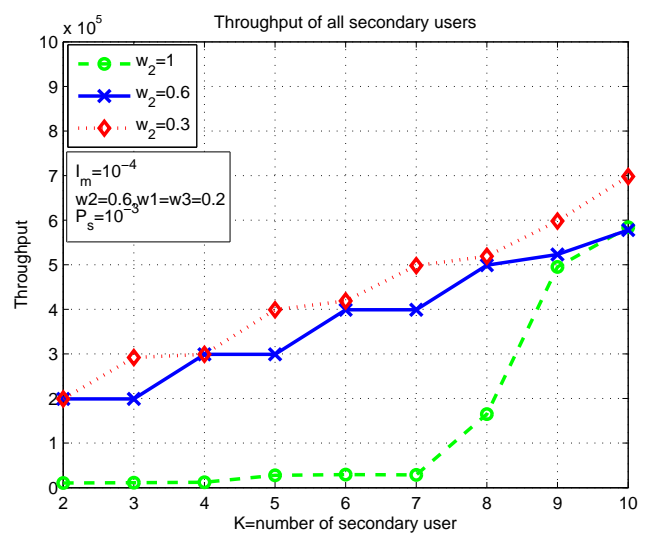

Figure 4.41: Throughput of (bps) all secondary users

In this case, $w_{2}=0.6 w_{1}=w_{3}=0.2$, which means more power control, less admission control and throughput guarantee. In comparison to Figure 4.2, the number of users is less by one when $K$ is 
under four and $K$ equals ten. Therefore less users are admitted when doing more power control since the second part of the objective function and constraint $\mathrm{C} 1$ limit the number of admitted users. The total power in this case shown in Figure 4.39 is $54 \%$ lower than that in Figure 4.4 which indicates that power $p_{k}$ is seriously limited when doing more power control, which leads to lower fval as Figure 4.40 shows compared to Figure 4.6. Moreover, slightly lower throughput is guaranteed as Figure 4.41 shows compared to Figure 4.8. In summary, in this case when $w_{2}$ equals 0.6 , much lower power is allocated, slightly lower throughput is guaranteed and lower function value is obtained.

Case 3: Throughput Guarantee $\left(w_{3}=\mathbf{0 . 6} w_{1}=w_{2}=\mathbf{0 . 2}\right)$

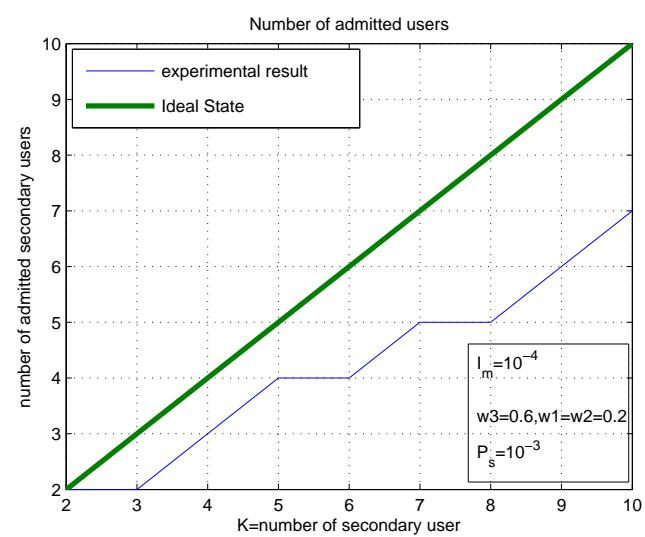

Figure 4.42: Number of admitted users

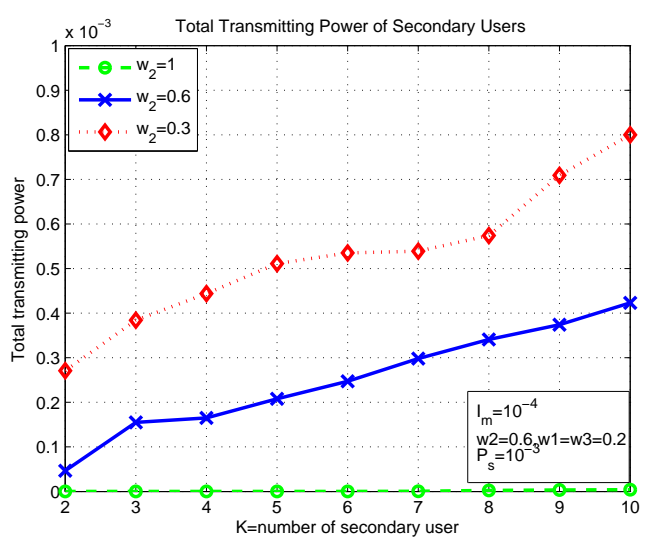

Figure 4.43: Total transmitting power (Watt) of all secondary users

In this case, $w_{3}=0.6 w_{1}=w_{2}=0.2$, which means that we focus on throughput guarantee. As the figures 4.42-4.45 show, results in this case are highly similar to that in previous case. The throughput is just $5 \%$ higher than the result in Figure 4.6 because we try to guarantee better throughput than the normal level in this case while reducing the function value, which is just slightly lower than the result in equal weight case as Figure 4.44 shows. The total power shown in Figure 4.43 is just as low as that in Figure 4.39 because we also want to reduce the value of the second part of the objective function. With lower total power, we must reduce the number of admitted users in order to guarantee better throughput for each secondary user, which is the reason why less number 


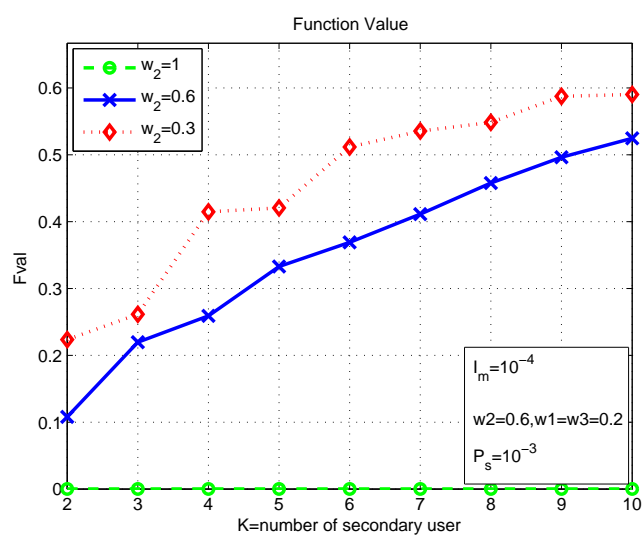

Figure 4.44: Function value

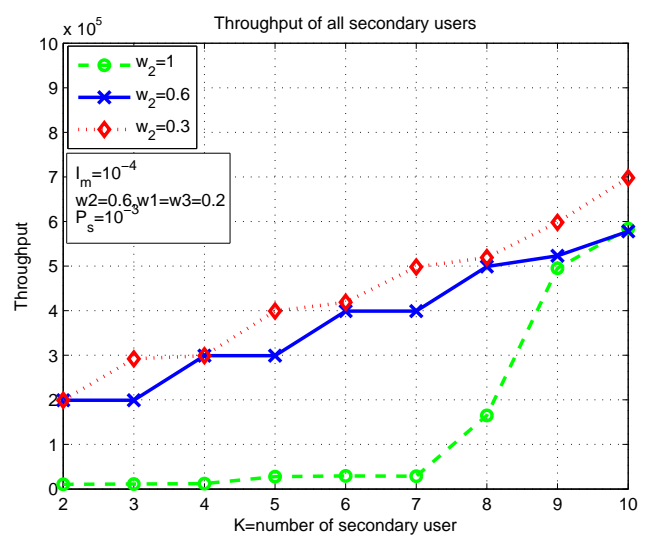

Figure 4.45: Throughput of (bps) all secondary users

of users are admitted in this case. The fval remains the same as that in Figure 4.40, because less users leads to a higher value of the first part of the objective function.

\subsection{Chapter Summary}

In this chapter, we first set particular values to all variables and constants. And we also explained how we calculate those values. Secondly, we run our MATLAB program for three different cases which are equal weights, unequal weights and single dominant weights, respectively. In each section, we also changed the value of $I_{m}$ and $P_{s}$ to see the difference. Thus, there are totally seven subsections introducing our results by plotting our results for number of admitted users, total transmitting power, function value and throughput of all secondary users.

Our numerical results indicate that more power is allocated and better throughput is guaranteed while the number of admitted users is increasing. However, as they increase, the function value increase steadily as well which means it is more and more difficult to reach the minimum function value, thus our minimizing objective is harder to achieve. The obtained results from the three cases including equal weights, unequal weights and single dominant weights indicate that we can change the weights to place more effort on a specific objective. 


\section{Chapter 5}

\section{Conclusions and Future Work}

\subsection{Conclusions}

We have presented an approach to solve the joint admission control and power allocation problem in a hospital environment based on cognitive radio. Specifically, a multi-objective non-convex mixed integer non-linear programming (MINLP) problem for wireless access in a hospital environment has been formulated in order to maximize the number of admitted secondary users and minimize transmit power while guaranteeing the throughput of all secondary users and satisfying the interference constraints for the protected and primary users.

To solve this MINLP problem, we proposed a standard branch and bound algorithm modified accordingly to find the optimal solution. We also coded a specific program in MATLAB using OPTI Toolbox to find the minimum function value and all related values such as total system power, throughput, and number of admitted users. Our numerical results indicate that more power is allocated and better throughput is guaranteed while the number of admitted users is increasing. However, as they increase, the function value increase steadily as well which means it is more and more difficult to reach the minimum function value, thus our minimizing objective is harder to achieve. The obtained results from the three cases including equal weights, unequal weights and single dominant weights indicate that we can change the weights to place more effort on a specific 
objective. For example, we can increase the value of the first weight to admit more secondary users. Different values of weights can be applied in real world to implement specific control objective as required by the system.

\subsection{Future work}

Based on this work, the following are open areas and potential research directions that might be interesting to pursue in the future

- In our system model, we only studied cognitive radio network in one floor of a hospital, similar network in multiple floors in a hospital building is a good future direction for investigation.

- We assumed that all kinds of users including primary users, secondary users and protected users are located at a fixed point, thus the distance between any two users are always fixed which makes it easier when we calculate the interference between two types of users. If all users are randomly located, it would be interesting to combine our problem with new placement optimization problem.

- We assumed uplink transmission in our analysis. This work can be extended to study the more complex interference between primary and secondary users for the case of uplink transmission.

- We used a basic branch and bound algorithm to solve our MINLP problem. A better hybrid algorithm, an integration of branch and bound and outer approximation algorithms can be applied to find more accurate values and better solutions.

- We only changed total source power and interference level to three different values when comparing their result difference. Another possibility is to use more variable values to see the large difference to be closer to reality. 
- we changed the value of weights for different objective, this control mechanism can be applied for developing different healthcare applications on different purpose. 


\section{Bibliography}

[1] P. Phond, "Dynamic wireless access methods with applications to ehealth services," 2009.

[2] G. Koutitas, "Power aware indoor wireless networks," Electronics Letters, vol. 46, no. 6, pp. 462-464, 2010.

[3] W. Tam and F. Lau, "Analysis of power control and its imperfections in cdma cellular systems," Vehicular Technology, IEEE Transactions on, vol. 48, no. 5, pp. 1706-1717, 1999.

[4] Z. Xia, W. Hao, I.-L. Yen, and P. Li, "A distributed admission control model for qos assurance in large-scale media delivery systems," IEEE Transactions on Parallel and Distributed Systems, vol. 16, no. 12, pp. 1143-1153, 2005.

[5] Y. He, W. Zhu, and L. Guan, "Optimal resource allocation for pervasive health monitoring systems with body sensor networks," IEEE Transactions on Mobile Computing, vol. 10, pp. $1558-1575$, nov. 2011.

[6] U. Varshney, "Pervasive healthcare and wireless health monitoring," Springer Science + Business Media, LLC, vol. 12, pp. 113 -127, jul. 2007.

[7] M. El Khaddar, H. Harroud, M. Boulmalf, M. Elkoutbi, and A. Habbani, "Emerging wireless technologies in e-health trends, challenges, and framework design issues," in 2012 International Conference on Multimedia Computing and Systems (ICMCS), pp. 440 -445, may 2012. 
[8] H. Alemdar and C. Ersoy, "Wireless sensor networks for healthcare: A survey," Computer Networks, vol. 54, no. 15, pp. $2688-2710,2010$.

[9] J. Ko, C. Lu, M. Srivastava, J. Stankovic, A. Terzis, and M. Welsh, "Wireless sensor networks for healthcare," Proceedings of the IEEE, vol. 98, pp. 1947 -1960, nov. 2010.

[10] Y. He, W. Zhu, and L. Guan, "Optimal resource allocation to provide qos guarantee in pervasive health monitoring systems," in 2011 IEEE International Conference on Multimedia and Expo (ICME), pp. $1-6$, july 2011.

[11] J. Stankovic, Q. Cao, T. Doan, L. Fang, Z. He, R. Kiran, S. Lin, S. Son, R. Stoleru, and A. Wood, "Wireless sensor networks for in-home healthcare: Potential and challenges." High Confidence Medical Device Software and Systems (HCMDSS) Workshop, 2005.

[12] Y. Zhang and H. Xiao, "Bluetooth-based sensor networks for remotely monitoring the physiological signals of a patient," IEEE Transactions on Information Technology in Biomedicine, vol. 13, pp. $1040-1048$, nov. 2009.

[13] L. Traver, C. Tarin, and N. Cardona, "Bandwidth resource management for neural signal telemetry," IEEE Transactions on Information Technology in Biomedicine, vol. 13, pp. 1083 -1084 , nov. 2009.

[14] B. Shrestha, E. Hossain, and S. Camorlinga, "Ieee 802.15.4 mac with gts transmission for heterogeneous devices with application to wheelchair body-area sensor networks," IEEE Transactions on Information Technology in Biomedicine, vol. 15, pp. 767 -777, sept. 2011.

[15] M. Fengou, G. Mantas, D. Lymberopoulos, N. Komninos, S. Fengos, and N. Lazarou, “A new framework architecture for next generation e-health services," IEEE Transactions on Information Technology in Biomedicine, vol. PP, no. 99, p. 1, 2012. 
[16] L. Skorin-Kapov and M. Matijasevic, "Analysis of qos requirements for e-health services and mapping to evolved packet system qos classes," International Journal of Telemedicine and Applications, vol. 2010, pp. 1- 18, jul. 2010.

[17] B. Lo and G.-Z. Yang, "Body sensor networks - research challenges and opportunities," in 2007 IET Seminar on Antennas and Propagation for Body-Centric Wireless Communications, pp. $26-32$, april 2007.

[18] K. M. Siassiakos and A. A. Lazakidou, Handbook of Research on Distributed Medical Informatics and E-Health. IGI Global.

[19] G. Cova, X. Huagang, G. Qiang, E. Guerrero, R. Ricardo, and J. Estevez, "A perspective of state-of-the-art wireless technologies for e-health applications," in IEEE International Symposium on IT in Medicine Education, 2009. ITIME '09., vol. 1, pp. 76 -81, aug. 2009.

[20] K. Kawamura, Y. Kobayashi, and M. Fujie, "Development of real-time simulation for workload quantization in robotic tele-surgery," in IEEE International Conference on Robotics and Biomimetics, 2006. ROBIO '06., pp. 1420 -1425, dec. 2006.

[21] S. Hasan, N. Siddique, and S. Chakraborty, "Femtocell versus wifi - a survey and comparison of architecture and performance," in 1st International Conference on Wireless Communication, Vehicular Technology, Information Theory and Aerospace Electronic Systems Technology, 2009. Wireless VITAE 2009., pp. 916 -920, may 2009.

[22] H. Ng, M. Sim, C. Tan, and C. Wong, "Wireless technologies for telemedicine," BT Technology Journal, vol. 24, pp. 130-137, 2006.

[23] A. Zvikhachevskaya, G. Markarian, and L. Mihaylova, "Quality of service consideration for the wireless telemedicine and e-health services," in Wireless Communications and Networking Conference, 2009. WCNC 2009. IEEE, pp. 1 -6, april 2009. 
[24] G. Markarian, L. Mihaylova, D. Tsitserov, and A. Zvikhachevskaya, "Video distribution techniques over wimax networks for m-health applications," IEEE Transactions on Information Technology in Biomedicine., vol. 16, no. 1, pp. 24-30, 2012.

[25] B. Lo and G.-Z. Yang, "Body sensor networks - research challenges and opportunities," in 2007 IET Seminar on Antennas and Propagation for Body-Centric Wireless Communications, pp. 26-32, 2007.

[26] U. Mitra, B. Emken, S. Lee, M. Li, V. Rozgic, G. Thatte, H. Vathsangam, D. Zois, M. Annavaram, S. Narayanan, M. Levorato, D. Spruijt-Metz, and G. Sukhatme, "Knowme: a case study in wireless body area sensor network design," Communications Magazine, IEEE, vol. 50, pp. $116-125$, may 2012.

[27] S. Weber, J. Andrews, X. Yang, and G. de Veciana, "Transmission capacity of wireless ad hoc networks with successive interference cancellation," IEEE Transactions on Information Theory, vol. 53, pp. 2799 -2814, aug. 2007.

[28] M. A. Khalid and K. K. Chai, "Performance comparison of ad hoc routing protocols in ehealth applications," Computer Science Journal, vol. 2, April 2012.

[29] J. Andrews, "Interference cancellation for cellular systems: a contemporary overview," Wireless Communications, IEEE, vol. 12, pp. 19 - 29, april 2005.

[30] O. Omeni, O. Eljamaly, and A. Burdett, "Energy efficient medium access protocol for wireless medical body area sensor networks," in 4th IEEE/EMBS International Summer School and Symposium on Medical Devices and Biosensors, 2007. ISSS-MDBS 2007., pp. 29 -32, aug. 2007.

[31] H. Fariborzi and M. Moghavvemi, "Architecture of a wireless sensor network for vital signs transmission in hospital setting," in International Conference on Convergence Information Technology, 2007., pp. $745-749$, nov. 2007. 
[32] P. Wang, Y. Yemini, D. Florissi, J. Zinky, and P. Florissi, "Experimental qos performances of multimedia applications," in INFOCOM 2000. Nineteenth Annual Joint Conference of the IEEE Computer and Communications Societies. Proceedings. IEEE, vol. 2, pp. 970-979 vol.2, 2000.

[33] A. Zvikhachevskaya, G. Markarian, and L. Mihaylova, "Quality of service consideration for the wireless telemedicine and e-health services," in Wireless Communications and Networking Conference, 2009. WCNC 2009. IEEE, pp. 1-6, 2009.

[34] D. Vouyioukas, I. Maglogiannis, and D. Komnakos, "Emergency m-health services through high-speed 3g systems: Simulation and performance evaluation," Simulation, vol. 83, pp. 329-345, Apr. 2007.

[35] L. Skorin-Kapov and M. Matijasevic, "Analysis of qos requirements for e-health services and mapping to evolved packet system qos classes," Int. J. Telemedicine Appl., vol. 2010, pp. 9:1-9:18, Jan. 2010.

[36] I. Martinez, J. Garcia, and E. Viruete, "Resources variability in m-health services: An adaptive method for qos control," in Consumer Communications and Networking Conference, 2008. CCNC 2008. 5th IEEE, pp. 829-833, 2008.

[37] T. Zahir, K. Arshad, A. Nakata, and K. Moessner, "Interference management in femtocells," IEEE Communications Surveys Tutorials, vol. PP, no. 99, pp. 1 -19, 2012.

[38] M. Yavuz, F. Meshkati, S. Nanda, A. Pokhariyal, N. Johnson, B. Raghothaman, and A. Richardson, "Interference management and performance analysis of umts/hspa+ femtocells," Communications Magazine, IEEE, vol. 47, pp. 102 -109, september 2009.

[39] N. Saquib, E. Hossain, L. B. Le, and D. I. Kim, "Interference management in ofdma femtocell networks: issues and approaches," Wireless Communications, IEEE, vol. 19, pp. 86 -95, june 2012. 
[40] K. Yeung and S. Nanda, "Channel management in microcell/macrocell cellular radio systems," IEEE Transactions on Vehicular Technology, vol. 45, pp. 601 -612, nov 1996.

[41] T. Zahir, K. Arshad, A. Nakata, and K. Moessner, "Interference management in femtocells," Communications Surveys Tutorials, IEEE, vol. 15, no. 1, pp. 293-311, 2013.

[42] I. E. Lamprinos, A. Prentza, E. Sakka, and D. Koutsouris, "Energy-efficient mac protocol for patient personal area networks," in 27th Annual International Conference of the Engineering in Medicine and Biology Society, 2005. IEEE-EMBS 2005., pp. 3799-3802, 2005.

[43] S. van der Burg, M. de Jonge, E. Dolstra, and E. Visser, "Software deployment in a dynamic cloud: From device to service orientation in a hospital environment," in ICSE Workshop on Software Engineering Challenges of Cloud Computing, 2009. CLOUD ’09., pp. 61-66, 2009.

[44] P. Phunchongharn, E. Hossain, and S. Camorlinga, "Electromagnetic interference-aware transmission scheduling and power control for dynamic wireless access in hospital environments," IEEE Transactions on Information Technology in Biomedicine, vol. 15, no. 6, pp. 890-899, 2011.

[45] S. Shah and Robinson, "Medical device technologies: who is the user?," Healthcare Technology and Management, vol. 9, no. 2, pp. 181-197, 2008.

[46] T. Rappaport, Wireless Communications: Principles and Practice. Upper Saddle River, NJ, USA: Prentice Hall PTR, 2nd ed., 2001.

[47] P.-C. Wang, J.-F. Tsai, W.-N. Ma, and C.-C. Lee, "An efficient global optimization approach for solving mixed-integer nonlinear programming problems," in 2010 40th International Conference on Computers and Industrial Engineering (CIE), pp. 1-4, 2010.

[48] P. Bonami, L. T. Biegler, A. R. Conn, G. Cornujols, I. E. Grossmann, C. D. Laird, J. Lee, A. Lodi, F. Margot, N. Sawaya, and A. Wchter, "An algorithmic framework for convex 
mixed integer nonlinear programs," Discrete Optimization, vol. 5, no. 2, pp. 186 - 204, 2008. ¡ce:title $i$ In Memory of George B. Dantzigi/ce:title $\underset{i}{.}$

[49] P. Bonami, "More branch-and-bound experiments in convex nonlinear integer programming," 2011.

[50] E. Kalvelagen, “Some minlp solution algorithms,” 2003.

[51] S. Hosseini-Khayat, P. Bahmanyar, E. Rahiminezhad, and M. Sawan, "Ultra-low power encryption engine for wireless implantable medical devices," in 2012 IEEE 55th International Midwest Symposium on Circuits and Systems (MWSCAS), pp. 150-153, 2012.

[52] L.-F. Tanguay and M. Sawan, "A fully-integrated 580x003bc;w ism-band frequency synthesizer for implantable medical devices," in International Symposium on Signals, Circuits and Systems, 2007. ISSCS 2007., vol. 1, pp. 1-4, 2007.

[53] O. Lauer, M. Riederer, N. Karoui, R. Vahldieck, E. Keller, and J. Fro?hlich, "Characterization of the electromagnetic environment in a hospital," in Electromagnetic Compatibility and 19th International Zurich Symposium on Electromagnetic Compatibility, 2008. APEMC 2008. Asia-Pacific Symposium on, pp. 474-477, 2008.

[54] “2010 guidelines for design and construction of health care facilities," 2010.

[55] A. C. Casas, "General public and workers exposure to high-frequency electric fields in spanish hospitals," Med Segur Trab (Internet), pp. 1-11, 2013. 